\title{
Measurements of Enhanced Near-Surface Turbulence under Windrows
}

\author{
Seth F. ZiPPEL, Ted MAKSym, AND MAlcolm SCUlly \\ Woods Hole Oceanographic Institution, Woods Hole, Massachusetts \\ PETER SutherLAND \\ IFREMER, Univ. Brest, CNRS, IRD, Laboratoire d'Océanographie Physique et Spatiale, IUEM, Brest, France \\ DANY DUMONT \\ Institut des sciences de la mer de Rimouski, Université du Québec à Rimouski, Rimouski, Québec, Canada
}

(Manuscript received 13 December 2018, in final form 13 November 2019)

\begin{abstract}
Observations of waves, winds, turbulence, and the geometry and circulation of windrows were made in a shallow bay in the winter of 2018 outside of Rimouski, Québec. Water velocities measured from a forwardlooking pulse-coherent ADCP mounted on a small zodiac show spanwise (cross-windrow) convergence, streamwise (downwind) velocity enhancement, and downwelling in the windrows, consistent with the view that windrows are the result of counterrotating pairs of wind-aligned vortices. The spacing of windrows, measured with acoustic backscatter and with surface imagery, was measured to be approximately twice the water depth, which suggests an aspect ratio of 1 . The magnitude and vertical distribution of turbulence measured from the ADCP are consistent with a previous scaling and observations of near-surface turbulence under breaking waves, with dissipation rates larger and decaying faster vertically than what is expected from a shear-driven boundary layer. Measurements of dissipation rate are partitioned to within, and outside of the windrow convergence zones, and measurements inside the convergence zones are found to be nearly an order of magnitude larger than those outside with similar vertical structure. A ratio of time scales suggests that turbulence likely dissipates before it can be advected horizontally into convergences, but the advection of wave energy into convergences may elevate the surface flux of TKE and could explain the elevated turbulence in the windrows. These results add to a limited number of conflicting observations of turbulence variability due to windrows, which may modify gas flux, and heat and momentum transport in the surface boundary layer.
\end{abstract}

\section{Introduction}

Turbulence in the ocean surface boundary differs dramatically from traditional boundary layer flow due to the presence of surface waves. In traditional wall layer flow, turbulent kinetic energy (TKE) dissipation rates $\varepsilon$ are expected to follow the relation $\varepsilon \propto u_{*}^{3} / z$, where $u *$ is the shear velocity defined $u_{*}=(\tau / \rho)^{1 / 2}$, and $z$ is the distance to the boundary. Here, $\tau$ is the stress at the boundary, and $\rho$ is the fluid density. Surface wave breaking, however, enhances dissipation rates beyond what is expected in the classic wall layer, providing a flux of TKE at the ocean surface (Agrawal et al. 1992; Craig and Banner 1994; Terray et al. 1996; Gerbi et al.

\footnotetext{
Corresponding author: Seth Zippel, szippel@whoi.edu
}

2009; Gemmrich 2010; Sutherland and Melville 2015). Just below the surface, there is a balance between the dissipation rate of TKE and the divergence of turbulent flux of TKE (Scully et al. 2016), which can be modeled using the gradient diffusion hypothesis (Craig and Banner 1994; Umlauf and Burchard 2003). In this wave-enhanced layer, TKE dissipation rates have been shown to scale as a power law decay (Terray et al. 1996),

$$
\frac{\varepsilon H_{s}}{F}=A\left(\frac{z}{H_{s}}\right)^{\lambda}
$$

where $H_{S}$ is the significant wave height, $F$ is the surface flux of TKE related to the energy lost from breaking waves, and $A$ and $\lambda$ are constants. Studies still disagree on the exact value of the decay exponent, although 
generally it is reported between $-2<\lambda<-1$ (Drennan et al. 1996; Terray et al. 1996; Gemmrich 2010; Sutherland and Melville 2015; Thomson et al. 2016). Very close to the surface $\left(z / H_{s}<0.6\right)$, Terray et al. (1996) proposed a layer of constant dissipation. This has been refuted by more recent studies which were able to measure close to the free surface, where instead of a constant layer, decay slopes are reported $-1<\lambda<-0.7$ (Gemmrich 2010; Sutherland and Melville 2015; Wang and Liao 2016). A number of studies have suggested that differences in reference frame (mean, or free surface) impact these estimates of decay slope (Gemmrich 2010; Thomson et al. 2016; Zippel et al. 2018). Craig and Banner (1994) provided an analytic solution for a balance between diffusive transport and dissipation,

$$
\frac{\varepsilon z_{0}}{F}=A\left(1+\frac{z}{z_{0}}\right)^{\lambda}
$$

where here $z_{0}$ is the surface roughness length, which can be thought of as being related to the length scale of injected turbulence. Zippel et al. (2018) confirmed that the roughness length $z_{0}$ scales with the wave height, and showed that the Craig and Banner (1994) analytic solution is consistent with decreased decay slopes close to the free surface.

Because of the rapid decay scale of wave-breaking turbulence, it affects processes at the air-sea interface (depths less than about $10 H_{s}$; Terray et al. 1996; Gerbi et al. 2009; Sutherland and Melville 2015) such as gas transfer, but may not generally be directly responsible for deepening oceanic mixed layers which can be at depths $\sim 100 \mathrm{~m}$. However, a second mechanism of waveenhanced turbulence affecting regions farther from the boundary has been proposed. The shear in surfacewave Stokes drift is hypothesized to rotate existing vertical vorticity (potentially seeded by wave breaking) into streamwise vorticity (Craik and Leibovich 1976). The resulting Langmuir circulations are often visually striking (Langmuir 1938). Coherent turbulent structures manifest as wave-aligned rows of bubbles (or other buoyant particulates), which gather in convergence zones that result from parallel counterrotating cells. Many large-eddy simulations (LESs) have reproduced similar coherent structures with the inclusion of the Craik-Leibovitch (CL) vortex force (Skyllingstad and Denbo 1995; McWilliams et al. 1997; Sullivan et al. 2007), and field experiments have measured downward jets, increased vertical velocity variance, and increased vertical velocity skewness under conditions where wave forcing (i.e., Stokes effects) exceeds wind and buoyant forcing (Smith 1996; Gargett and Wells 2007; Scully et al.2015). The relative importance of wind and wave forcing can be evaluated through the turbulent Langmuir number,

$$
\mathrm{La}_{t}^{2}=\frac{u_{*}}{U_{s 0}}
$$

where $U_{s 0}$ is the surface Stokes drift (McWilliams et al. 1997). Modified Langmuir numbers have been suggested (e.g., Kukulka and Harcourt 2017) because of the sensitivity of $U_{s 0}$ to the high frequency waves. Because of the tight relationship between short ocean waves and wind stress, $\mathrm{La}_{t}$ does not vary widely over realistic ocean conditions.

Li and Garrett (1995) introduced the Hoenikker number (Ho), which relates the relative importance of buoyancy forcing that drives thermal convection to the wave forcing that drives the CL mechanism,

$$
\mathrm{Ho}=\frac{-2 \alpha g Q}{\rho_{w} C_{p, w} k_{p} U_{s 0} u_{*}^{2}},
$$

where $\alpha=2.3 \times 10^{-4}{ }^{\circ} \mathrm{C}^{-1}$ is the coefficient of thermal expansion, $C_{p, w} \approx 4100 \mathrm{~J} \mathrm{~kg}^{-1}{ }^{\circ} \mathrm{C}^{-1}$ is the specific heat of water, and $Q$ is the surface heat flux. Values of $\mathrm{Ho} \geq 1$ indicate buoyant forces dominate over wave forces, while values of Ho $\leq-1$ indicate stability shuts down wave-induced turbulent motions.

Previous studies have documented the aspect ratio and velocity scales of windrows in deep and shallow water. Plueddemann et al. (1996) used Doppler sonar in deep water to measure the near surface convergent velocities, convergence cell spacing, and the resulting bubble plume depth. They found that convergent velocities scaled well with $\left(u_{*} U_{s 0}\right)^{1 / 2}$. Gargett and Wells (2007) made measurements of Langmuir cells in shallow water under forcing from intermediate depth waves $(0.05<d / L<0.5$, with $d$ being water depth and $L$ being wavelength) using a 5-beam ADCP. They found three-dimensional velocity fields were similar to those in deep water, consistent with the theory of counterrotating parallel vortex pairs, with strong downwelling velocities in the convergences and weaker upwelling in between convergences. In contrast to deep water, Gargett and Wells (2007) found cell aspect ratios were stretched horizontally $\left(L_{x} / d>2\right)$, and turbulent Langmuir numbers were larger than in deep water $\left(\mathrm{La}_{t} \sim 0.7\right)$ due to the bathymetric effects on Stokes drift. Scully et al. (2015) presented measurements from an ADCP and a turbulence tower in Chesapeake Bay, showing increased vertical TKE, decreased turbulence anisotropy, and negative vertical velocity skewness when $\mathrm{La}_{t}<0.5$. While they note that these flow characteristics appear to be the result of the CL vortex force, a second study on the same dataset 
TABLE 1. Overview of sampling conditions. Wind speeds are adjusted from the $75-\mathrm{cm}$ measurement height to the 10 -m equivalent using the estimated friction velocity. Reported values are the median measured values during deployment, rounded to the nearest tenth. Streak spacing also reports the standard deviation. Bubble streaks were not seen on 7 Mar.

\begin{tabular}{|c|c|c|c|c|c|c|c|c|c|}
\hline Date & $\begin{array}{l}\text { No. of } \\
\text { transects }\end{array}$ & $\begin{array}{c}\text { Wind } \\
\text { speed }\left(\mathrm{m} \mathrm{s}^{-1}\right)\end{array}$ & $\begin{array}{c}\text { Water } \\
\text { temperature }\left({ }^{\circ} \mathrm{C}\right)\end{array}$ & $\begin{array}{c}\text { Air } \\
\text { temperature }\left({ }^{\circ} \mathrm{C}\right)\end{array}$ & $\begin{array}{c}\text { Depth } \\
\text { range }(\mathrm{m})\end{array}$ & $H_{s}(\mathrm{~m})$ & $\begin{array}{c}\text { Streak } \\
\text { spacing }(\mathrm{m})\end{array}$ & $\mathrm{La}_{t}(-)$ & $c_{p} / u_{*, a}(-)$ \\
\hline $4 \mathrm{Mar}$ & 11 & 8.9 & 1 & 2.6 & $2-4$ & 0.3 & $6.9 \pm 3.7$ & 0.59 & 7.2 \\
\hline $6 \mathrm{Mar}$ & 8 & 5.9 & 1 & 2.6 & $1.5-3.5$ & 0.3 & $8.1 \pm 6.7$ & 0.57 & 7.7 \\
\hline 7 Mar & 19 & 4.0 & 0.8 & 2.3 & $1.5-3.9$ & 0.2 & - & 0.60 & 7.6 \\
\hline 8 Mar & 17 & 7.8 & 0.6 & 1.4 & $1.5-4.5$ & 0.3 & $6.7 \pm 3.6$ & 0.58 & 7.5 \\
\hline 9 Mar & 15 & 8.0 & 0.1 & 3.1 & $1.5-4.5$ & 0.6 & $6.7 \pm 4.5$ & 0.58 & 6.9 \\
\hline
\end{tabular}

(Scully et al. 2016) showed that the Strokes drift production did not significantly contribute to the TKE equation, which was well balanced by pressure work (turbulent transport) and dissipation (consistent with wave breaking).

To the authors' knowledge, only two field studies have documented the horizontal variability of turbulence when windrows are present. Thorpe et al. (2003) used microstructure probes on an AUV that transected windrows, finding turbulence was enhanced in the convergences by a factor of 2 . In the same dataset, Thorpe et al. (2003) reported turbulence levels that were in agreement with the law of the wall scaling, in contrast to recent findings that show enhanced near surface dissipation under breaking waves. Gemmrich (2012) reported measurements of turbulence in bubble convergences made with high resolution acoustics from a tethered drifter. However, Gemmrich (2012) found turbulence levels in the convergences were suppressed, and showed that this decrease in turbulent dissipation was well correlated with a buoyancy frequency that included the void fraction of entrained air bubbles. Gemmrich (2012) suggested that two near surface layers exist to reconcile their results with those of Thorpe et al. (2003): 1) the very near the surface where turbulence scales vertically as in Terray et al. (1996), but turbulence is suppressed in convergences, and 2) the underlying layer where the results from Thorpe et al. (2003) apply, and turbulence scales as law of the wall, but with local enhancement in the bubble convergences.

Here, we present measurements of the geometry, near-surface circulation, and turbulence associated with windrows formed in a shallow fetch-limited environment. These measurements add to the small number of studies that document spatial heterogeneity in surface turbulence associated with coherent structures. Measurements, data processing, and the field site are presented in section 2. Results, including windrow spacing, windrow circulation, and turbulence measurements are shown in section 3 . The implications of the measurements are discussed in section 4 , including a suggested mechanism for the turbulence enhancement. A summary is provided in section 5 .

\section{Methods}

Deployments were made in association with the BicWin project (Sutherland and Dumont 2018) in Parc National du Bic in the Lower St. Lawrence Estuary (Zippel et al. 2019). Measurements of winds, waves, turbulence, and currents from a Surface Wave Instrument Float with Tracking (SWIFT) drifter (Thomson 2012), a small Lagrangian float designed to sample close to the free surface, were complemented with zodiac measurements of water depth, currents, and turbulence. In addition, images of the sea surface were made from a zodiac-mounted GoPro, and a DJI Mavic Pro quadcopter. Measurements span 5 days in early March 2018, where offshore winds, wave breaking, and the accumulation of bubbles in wind-aligned rows were seen during four deployments. Measurements were taken near high slack tide, when tidal currents were small (typically less than $0.1 \mathrm{~m} \mathrm{~s}^{-1}$ ) and water depths were optimal for launching the zodiac (typically between 1.5 and $4.5 \mathrm{~m}$ ). Local fetch-limited wind waves (typically growing to $L \sim 3$ m over the length of the embayment) were accompanied by longer waves $(\sim 5 s)$ refracting into the embayment from the greater estuary. These longer waves were not visible by eye, but accounted for a large fraction of the significant wave height measured by the SWIFT buoy. Combined latent and sensible heat fluxes were relatively small $|Q|<30 \mathrm{~W} \mathrm{~m}^{-2}$, as the atmosphere and ocean were at similar temperatures. An overview of deployment dates and environmental conditions are presented in Table 1.

The zodiac was equipped with two $1-\mathrm{MHz}$ Nortek Signature AD2CPs. One AD2CP was oriented downward to measure mean currents and water depths (denoted LR hereafter), the other was equipped with HR (pulse coherent) firmware, and mounted on an L-frame looking forward, such that the 5 acoustic beams were ahead of any pressure wake effects (denoted HR hereafter, 


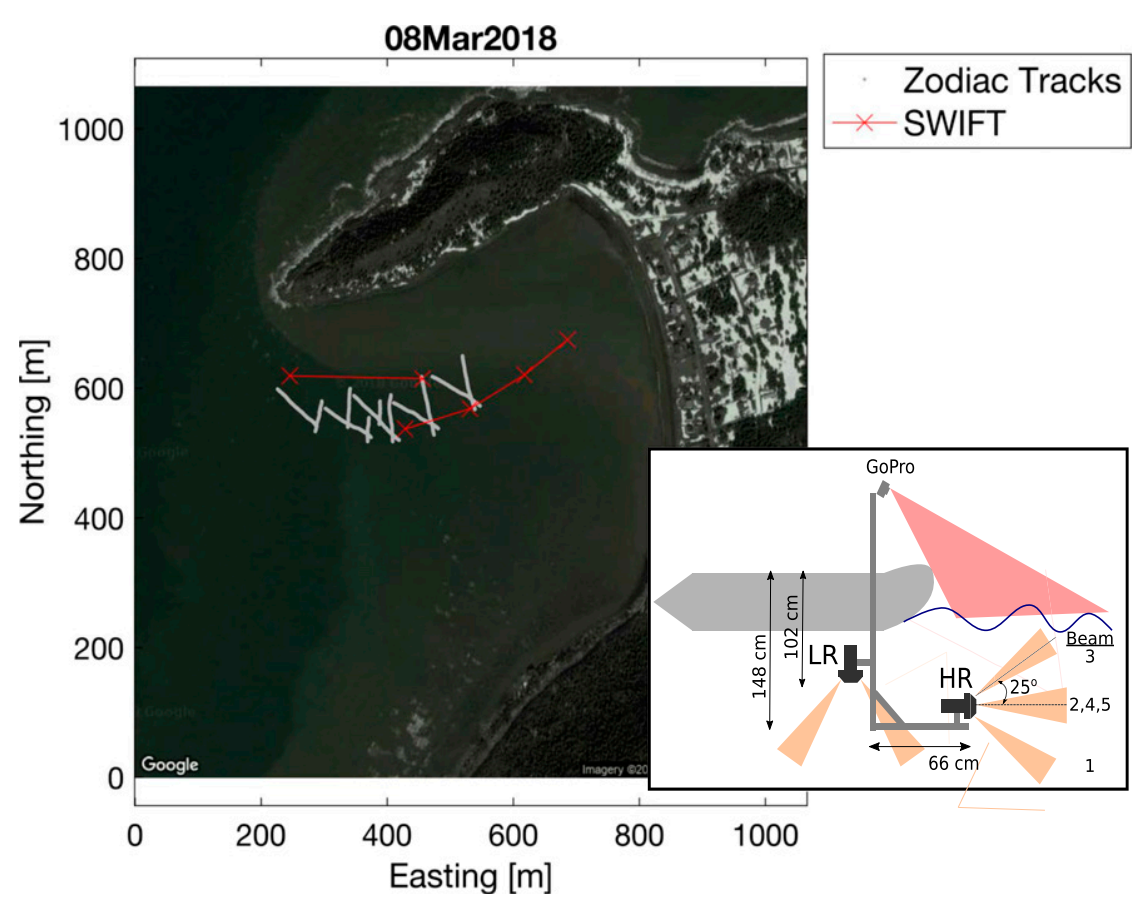

FIG. 1. Zodiac transects (gray) from 8 Mar 2018 are shown with SWIFT drift tracks (red). The inset shows a schematic of the forward-mounted HR Nortek Signature and the GoPro camera (not to scale). Wind was from the northeast, such that the drifters slowly moved toward the bay mouth.

see inset, Fig. 1). The forward mounted (HR) Nortek AD2CP was configured to measure velocities at $4 \mathrm{~Hz}$, with $2-\mathrm{cm}$ velocity bins out to a range of $2 \mathrm{~m}$. The Nortek Extended Velocity Range (EVR) was enabled, which uses the new Multi Correlation Pulse-Coherent (MCPC) processing, greatly increasing the velocity range of pulse coherent measurements before phase wrapping occurs (Shcherbina et al. 2018). In the past, HR measurements have been restricted to drifting platforms or low flow conditions to avoid the phase wrapping ambiguity inherent in the HR processing method. However, the new MCPC EVR extends this phase wrapping range such that measurements from a moving zodiac were possible with mean drive speeds near $40 \mathrm{~cm} \mathrm{~s}^{-1}$ in addition to boat heave and pitch motions.

Zodiac measurements were collected in $\sim 50$-m-long, cross-windrow transects. The orientation of the zodiac was kept perpendicular to the visible windrows, however due to currents and windage on the zodiac, transects over ground angled in the downwind direction. Between transects, the zodiac was driven upwind to avoid contamination by turbulence created by the propeller in the previous transect. A Qstarz BT-Q1000ex GPS recorded $10-\mathrm{Hz}$ position (from which velocity was inferred) for the zodiac, and a GoPro camera mounted $\sim 1 \mathrm{~m}$ above the surface recording at $1 \mathrm{~Hz}$ was able to visualize bubble accumulation on the surface. Coincident with the cross-windrow zodiac transects, the SWIFT drifter was deployed at the upwind extent of the embayment, and freely drifted toward the mouth of the embayment, where it was recovered and reset $(\sim 100 \mathrm{~m}$ from the shoreline). Each day consisted of 2-4 total drifts while tidal elevations remained high enough for safe zodiac operations. The DJI Mavic Quadcopter took surface imagery, but flight time was limited by battery life, and the sampling scheme (image type and altitude) was not consistent across all deployment days. Still, it was possible to identify the wavelength of the short waves (see section 2c) on all days, and the spacing of the windrows on two days where the quadcopter was flown at lower altitudes, and higher resolution imagery was used (see section 2b). An example deployment showing zodiac transects and SWIFT drift locations is shown in Fig. 1, and an example quadcopter image is shown in Fig. 2.

\section{a. Friction velocities and TKE fluxes}

Wind speed was measured at $75-\mathrm{cm}$ height on the SWIFT drifter with a $10-\mathrm{Hz}$ RM Young sonic anemometer. The buoy motion was removed from the wind velocity time series using the internal motion package (Microstrain 3DM-GX3-35 recording at 25 Hz) following the method of Edson et al. (1998). Wind stress 


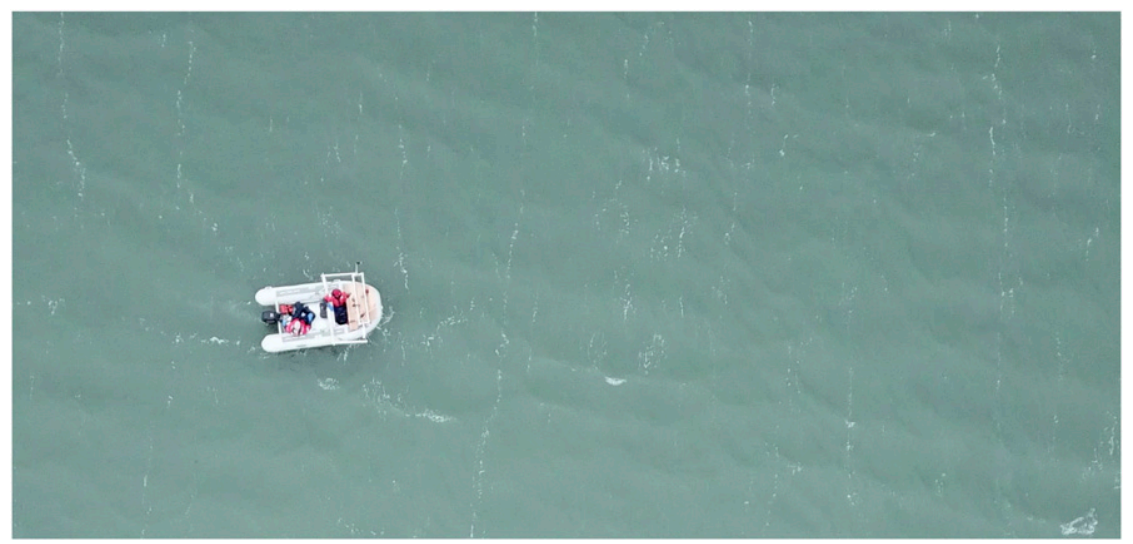

FIG. 2. Surface image take from the quadcopter showing the bands of wind/wave aligned bubbles. The image has been cropped and rotated such that the wind/wave direction of propagation is toward the bottom of the page.

was estimated with the inertial method (Large and Pond 1981; Yelland et al. 1994; Thomson et al. 2013). Vertical velocity spectra $E_{\mathrm{ww}}(f)$ were estimated for each 10-min segment with 512-point windows, and 50\% overlap. Airside TKE dissipation rates were estimated from

$$
\varepsilon_{a}=\left[\frac{\left\langle E_{\mathrm{ww}}(f) f^{5 / 3}\right\rangle}{K\left(\frac{U}{2 \pi}\right)^{2 / 3}}\right]^{3 / 2},
$$

where \langle\rangle represents an average over $1<f<4 \mathrm{~Hz}$ (representative of the inertial subrange), $K=0.55$ is a constant (Yelland et al. 1994), and $U$ is the horizontal advective velocity. The friction velocity was then estimated assuming a log boundary layer using

$$
u_{*, a}=\left(\kappa \varepsilon_{a} z\right)^{1 / 3},
$$

with $z=0.75 \mathrm{~m}$ being the measurement height, and $\kappa=0.4$ being von Kármán's constant. The equivalent waterside friction velocity is then $u_{*}=u_{*, a} \sqrt{\rho_{a} / \rho_{w}}$. The use of the inertial method is justified in the similarity of ocean and air temperatures (Table 1), indicating stability effects were relatively small. The inertial dissipation method can be biased low due to the energy transfer to the wave field (Janssen 1999), which results in a deficit of atmospheric TKE dissipation rate at heights $k z<2$. Due to the short wavelengths seen in this study $(L<3 \mathrm{~m})$, the measurements are typically above the region where these energy transfers are significant (e.g., Hare et al. 1997), although it is possible stresses are underestimated by $1 \%-10 \%$.

The resulting friction velocities are in good agreement with a bulk drag law with a 0.75 -m drag coefficient $C_{d, a}=$ $1.3 \times 10^{-3}$ (equivalent $10-\mathrm{m} C_{d, a} \approx 1 \times 10^{-3}$ ). The surface flux of TKE $F$ is calculated as $c_{e} u_{*}^{2}$ (Gemmrich et al. 1994), with $c_{e}=1.5 \mathrm{~m} \mathrm{~s}^{-1}$, which is within the range of values suggested in the literature (e.g., Terray et al. 1996, their Fig. 6 with young waves and $c_{p} \sim 3 \mathrm{~m} \mathrm{~s}^{-1}$ for short fetch waves). Other surface TKE flux formulations yield similar results for the range of wind speeds in this study, including $\alpha u_{*}^{3}$ with $\alpha=100$ (Craig and Banner 1994; Burchard 2001; Jones and Monismith 2008).

Estimates of the total latent and sensible heat flux were made from the sonic anemometer using the correlation of sonic temperature and vertical velocity $\left\langle T_{s}^{\prime} w^{\prime}\right\rangle$ (Liu et al. 2001). We estimate the range of values are likely limited by $|Q|<30 \mathrm{~W} \mathrm{~m}^{-2}$. These measurements include low-frequency noise related to the motion of the measurement platform, which was not fully removed by the motion correction.

Bottom friction velocity was estimated using a depthaveraged velocity calculated from the downward oriented AD2CP. Raw measured velocities were corrected for the speed over ground measured using the GPS data, and referenced to the bottom elevation seen in the backscatter data. Bottom stress was estimated using this depth-averaged velocity (representative of the bottom boundary) and a drag coefficient $u_{* \text {,bot }}=U \sqrt{C_{d, \text { bot }}}$, with $C_{d \text {,bot }}=2.5 \times 10^{-3}$. This estimate is likely biased high due to compass offsets resulting in incomplete removal of the zodiac speed over ground. Still, velocities measured with the downward oriented $\mathrm{AD} 2 \mathrm{CP}$ were of similar amplitude and direction to those measured at the surface by the SWIFT drifter (typically within a few $\mathrm{cm} \mathrm{s}^{-1}$ ).

\section{b. Streak identification}

Bubbles are identified in GoPro images using an adaptive histogram method (Otsu 1979), implemented using the MATLAB "imbinarize" function. Pixels associated with the zodiac and instrument frames (which are visible in the image) are removed from further 

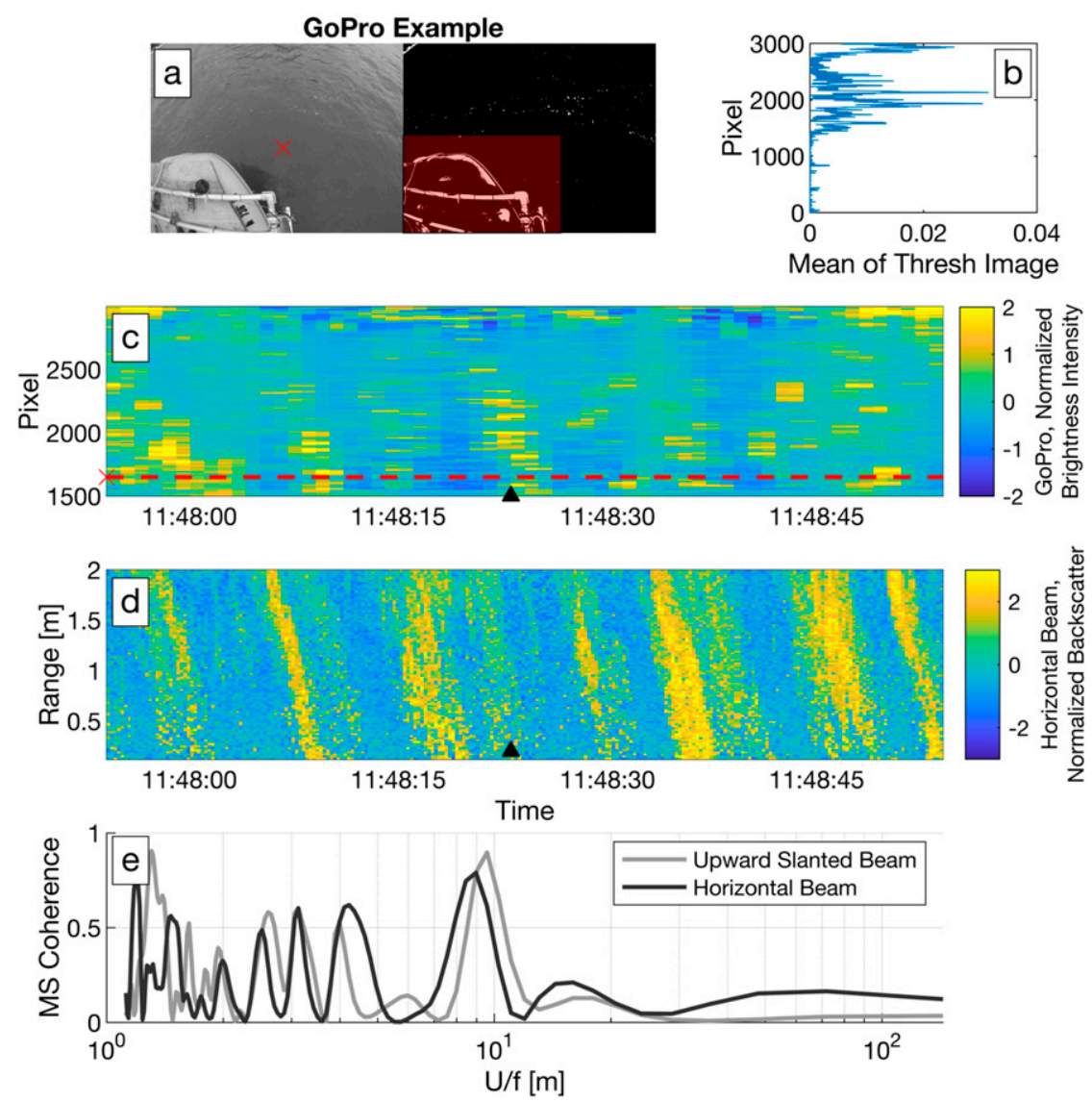

FIG. 3. (a) An example GoPro image and its thresholded binary image, with a red " $\times$ " marking the approximate location of the AD2CP. (b) The averages across each pixel row of the binary (threshold) image, denoted brightness intensity, where a corresponding increase in the brightness intensity relates to the streak of bubbles in the center of the GoPro image. Here, larger pixel values are more distant from the $\mathrm{AD} 2 \mathrm{CP}$, but are not rectified into distances. (c) The time series of brightness intensity, with (d) a corresponding example of the normalized acoustic backscatter. A black triangle denotes the time where the image in (a) was taken. (e) A peak in the magnitude squared coherence between the thresholded bubble images and the acoustic backscatter at approximate length scales of 7-10 $\mathrm{m}$ (frequencies converted to distance using the boat speed over ground). Data in this figure are from 8 Mar 2018.

processing with an image mask. Last, each row of pixels is averaged in the along-streak direction to give a pixel threshold intensity in the across-streak direction for each image frame. The image pixels are not rectified, as the camera orientation was not recorded. However, each image gives an estimate of bright pixels associated with bubbles in distance (pixel row) from the zodiac. An example of the GoPro methods, are shown in Fig. 3 with the collocated, forward looking AD2CP backscatter.

Regions of bubble accumulation are inferred from forward looking (HR) AD2CP backscatter. Profiles of backscatter are range-corrected by subtracting the mean at each $2 \mathrm{~cm}$ range bin, and normalized with the standard deviation of the bin. Profiles are categorized to be inside, or outside of convergence zones (where backscatter is elevated) as follows. A threshold is applied to the normalized backscatter profiles (Otsu's method implemented with MATLAB's imbinarize function, as with the GoPro data), and profiles are labeled as inside the high backscatter regions (referred to from now on as convergence zones, or streaks) if a profile of 95 bins has 35 or more thresholded values. A profile is labeled as outside these regions if it has fewer than 15 thresholded values. Profiles with between 15 and 35 thresholded values are not classified. The number of threshold bins used to classify each profile is heuristic, but was determined considering acoustic variations in the absence of the streaks, and the streak widths. The results were not significantly modified if the threshold values were modified by \pm 5 bins. 
Spacing between convergence zones is found by projecting normalized AD2CP backscatter data into realworld spatial coordinates using the GPS location and beam orientations. These scattered points are averaged into an along-track (cross-windrow) 1D coordinate, and smoothed with a moving average filter with equivalent spatial width of $1 \mathrm{~m}$. A range of smoothing widths between 0.25 and $1.5 \mathrm{~m}$ was used, and a $1-\mathrm{m}$ width was determined to reduce false peak identification due to noise without a significant bias against the existing smaller scales. Streaks are identified using MATLAB's "findpeaks" function, with a minimum prominence and peak height of 0.5 and 0.25 (units of normalized backscatter), respectively. These parameters were found to reduce identification of local noise. Last, occasional acoustic interference between the SWIFT ADCP and the zodiac $\mathrm{AD} 2 \mathrm{CP}$ was seen in brief intervals when the zodiac came in close proximity to the drifter. These spikes were accounted for by excluding peaks with a prominence greater than 3 . This process was repeated for both the HR forward looking AD2CP and for the downlooking LR AD2CP. Because the instruments' beams span the upper (HR) and lower (LR) parts of the water column, the comparison between the two estimates (HR and LR) can give information on the vertical structure of cell spacing.

Bubble streaks were visible in video collected from the quadcopter, and data of sufficient quality for quantitative analysis were collected on 8 and 9 March. Visible breaking was not seen on $7 \mathrm{March}$, and the imagery on 4 and 6 March was taken from a higher altitude reducing the visibility of bubbles (and thus the ability to easily identify spacing). On 8 and 9 March, the $4 \mathrm{~K}$ video data were processed as follows: a stationary segment of each video file was used for analysis by finding low variance in the quadcopter altitude, speed, and gimbal pitch and yaw. Bright pixels associated with bubbles were isolated in each frame using MATLAB's imbinarize function (in the same fashion as the GoPro imagery), and $30 \mathrm{~s}$ of thresholded video frames were averaged such that bubbles associated with individual breaking waves could be distinguished from the persistent bubble streaks. Thresholded values in individual frames associated with the zodiac or the drifter were identified as regions with more than 100 connected pixels, and removed. Streak spacing is found in the 30 -s averages using MATLAB's findpeaks function on 300-pixel (cross-streak) average slices, which have been smoothed with a $1-\mathrm{m}$-wide moving average filter. The spacing for each $30-\mathrm{s} 2 \mathrm{D}$ averaged image is taken as the mean of the difference in peak location, which are converted from pixels to meters using the quadcopter altitude, and camera pixel size and focal length.

\section{c. Stokes drift estimates}

Wave measurements from the SWIFT drifter were complemented by quadcopter video to better capture the short, fetch-limited waves at frequencies higher than resolved by the drifter (SWIFT reports frequencies up to $0.5 \mathrm{~Hz}$ ). These short waves significantly contribute to the surface Stokes drift. While optical imagery of the sea surface has been used for much more detailed analysis of the wave field (e.g., Plant et al. 2008), for this study we restricted analysis of quadcopter imagery to estimates of the peak wavelength of the short, locally generated waves. Two-dimensional Fourier transforms of video frames were averaged, and peaks were identified using the "FastPeakFind" function (available on the MATLAB file exchange). Projected pixel sizes were estimated using the camera focal length, sensor size, and altitude, and the spatial frequencies of the peak of the 2D image Fourier transform were taken as the peak wavenumber of the fetch limited waves $k_{p}$. These wavenumbers were in good agreement with the fetch laws (Kahma and Calkoen 1992). The peak wavenumber, along with the water depth, and wind speed and friction velocity taken from nearby zodiac/SWIFT measurements were used to estimate the Elfouhaily et al. (1997) 2D spectrum $\Psi\left(k_{x}, k_{y}\right)$ for each video file. Last, surface Stokes drift is estimated from the 2D spectrum with

$$
\mathbf{U}_{s 0}=\int_{-\infty}^{\infty} \int_{-\infty}^{\infty} \sigma(k) \mathbf{k} \Psi\left(k_{x}, k_{y}\right) \frac{\cosh [2 k(z+d)]}{\sinh ^{2}(k d)} d k_{x} d k_{y},
$$

where $z=0$, and here $\sigma(k)^{2}=\left(g k+\Gamma k^{3} / \rho\right) \tanh (k d)$ is the linear dispersion relation (including surface tension effects, with surface tension $\Gamma=0.074 \mathrm{~N} \mathrm{~m}^{-1}$ ). These estimates of surface Stokes drift include contributions from short waves not considered in previous studies, which we justify with the proximity of the measurements to the surface, and the shallow depths. For reference, if only wavelengths equal to or longer than the minimum depth of the experiment (about $1 \mathrm{~m}$ ) are considered in Stokes drift estimates, the resulting $\mathrm{La}_{t}$ is increased by $\sim 20 \%$ (approximately from 0.55 to 0.73 ).

\section{d. Conditional averaged velocity}

Velocities measured by the AD2CP were conditionally averaged to estimate the circulation associated with the windrows. Windrows (identified as in section $2 b$ ) at each range bin were used to normalize distances in the spanwise (cross-windrow) direction (which inherently assumes a constant boat speed across these distances). Velocities for each zodiac transect were de-meaned, 
mapped to this nondimensional spanwise coordinate, interpolated onto a regularly spaced spanwise grid, and smoothed with a loess filter. Velocities from each transect were normalized by the surface friction velocity, and averaged across all transects to achieve an experiment average circulation. Surface gravity wave and platform motions are periodic and have a phase that is uncorrelated to the windrows, and thus these otherwise large velocity signals average to zero with a sufficient number of observations. Because the relative orientations of the zodiac to the individual windrows were unknown, velocities were kept in beam coordinates during averaging, before transforming into streamwise, spanwise, and vertical components. Alternating track directions changed the sign of the downwind (streamwise) velocity component, such that the relative track direction to the wind direction determined the sign of the spanwise component. The conditionally averaged beam velocities were converted to three components of velocity using the beam spread $\theta=25^{\circ}$, and standard $\mathrm{AD} 2 \mathrm{CP}$ transformations, and then an average was taken across range bins to make a spanwise average of the three velocity components. The upward slanted beam was restricted to the first 50 range bins to minimize the vertical extent of the measurements. Therefore, the vertical components represents an average over depths $0.3<z<0.8 \mathrm{~m}$, with the spanwise and streamwise components at $z \sim 0.8 \mathrm{~m}$. Because of the uncertainties in relative beam orientations to the windrows, and the averaging and filtering applied to this velocity product, the reported velocity magnitudes and gradients are likely biased small.

\section{e. Turbulence}

TKE dissipation rate profiles were estimated using a second-order structure function method. This approach has the advantage of being robust toward platform motions which affect velocity profiles uniformly in time. The structure function approach has been used by numerous other surface turbulence studies (e.g., Gemmrich 2010; Thomson et al. 2012; Zippel et al. 2018), and easily handles missing data (as opposed to spectral methods). A phase-unwrapping method was applied (similar to the Ito unwrapping scheme in Shcherbina et al. 2018), where velocities were checked for jumps near integer multiples of the wrapping velocity. Suspect points were then moved $\pm n$ wrapping velocities according to the number of suspected wraps $n$. Data within $10 \mathrm{~cm}$ of the surface are not used due to potential side-lobe effects. Only AD2CP pulse correlations greater than 75 , and amplitudes greater than 30 counts were used. Velocities not meeting these criteria were omitted from analysis. These correspond to similar values used in previous studies (Elgar et al. 2001; Thomson et al. 2012), although it has been noted that only using correlations greater than 75 may be overly restrictive (Rusello 2009).

A centered, or two-sided, second-order structure function $D(r, d r)$ was used for estimating TKE dissipation rates, which is defined as

$$
D(r, d r)=\left\langle\left[u^{\prime}(r-d r / 2, t)-u^{\prime}(r+d r / 2, t)\right]^{2}\right\rangle,
$$

where $u^{\prime}(r, t)$ is a turbulent velocity at range $r$ from the instrument taken at time $t, d r$ is a separation distance between bins, and \langle\rangle represents an average across measurements in time. The expected relation of the second-order structure function to TKE dissipation rate $\varepsilon$ is

$$
D(r, d r)=C_{v} \varepsilon^{2 / 3} d r^{2 / 3},
$$

where $C_{v}=2.1 \pm 0.1$ is a constant (Wiles et al. 2006). This relation is expected to hold over the inertial subrange, where turbulent motions are controlled primarily by inertial effects.

Measured velocities contain nonturbulent components due to Doppler noise $\sigma_{u}$, wave effects $\tilde{u}$, and platform motion (translation $u_{\text {trans }}$ and rotation $u_{\text {rot }}$ ), such that the mean removed measured velocity can be expressed $u_{\text {meas }}=u^{\prime}+\sigma_{u}+\tilde{u}+u_{\text {trans }}+u_{\text {rot }}$. Platform translational motion is uniform across velocity bins, and is thus removed by the subtraction in Eq. (8). However, Doppler noise, wave orbital velocities, and platform rotations may add nonturbulent contributions to the structure function estimated from measurements. Under the assumption that these three nonturbulent motions in the measured velocities are uncorrelated, the resulting measured structure function is

$$
\begin{aligned}
D_{\text {meas }}(r, d r) & =\left\langle\left[u_{\text {meas }}(r-d r / 2, t)-u_{\text {meas }}(r+d r / 2, t)\right]^{2}\right\rangle \\
& =2 \sigma_{u}^{2}+D(r, d r)+\tilde{D}(r, d r)+D(r, d r)_{\text {rot }},
\end{aligned}
$$

where $\sigma_{u}^{2}$ is the variance due to Doppler noise, $\tilde{D}(r, d r)$ is the wave contribution, and the rotational motion contribution should scale $D(r, d r)_{\text {rot }} \sim d r^{2}$, since rotational velocities have the form $\mathbf{u}_{\mathrm{rot}}^{2}=(\mathbf{r} \times \omega)^{2}$. Recently, Scannell et al. (2017) suggested the wave contribution to the structure function $\tilde{D}(r, d r)$ also should scale as $d r^{2}$ (an investigation of the wave effects on the second-order structure function is presented in the appendix, including the effects of wave phase). The desired turbulent contributions to the measured structure function can be 
separated through a multiple linear regression (Scannell et al. 2017),

$$
D_{\text {meas }}(r, d r)=N(r)+A(r) d r^{2 / 3}+B(r) d r^{2},
$$

where $N(r), A(r)$, and $B(r)$, are the regression coefficients. To minimize the contributions from rotational and wave effects which scale as $d r^{2}$, a max separation distance of $d r_{\max }=1.2 \mathrm{~m}$ is imposed. TKE dissipation rates are then estimated at range $r$ from the regression coefficient $A(r)$,

$$
\varepsilon(r)=\left[\frac{A(r)}{C_{v}}\right]^{3 / 2}
$$

Last, the beam position is mapped to the water depth using the AD2CP depth and orientation, such that $\varepsilon(z)$ is determined from $\varepsilon(r)$. As an additional quality control metric, noise offsets $N(r)$ are qualitatively checked to be within a range of noise values expected for the instrument [exact noise estimates are nontrivial, e.g., Zedel et al. (1996), but are typically $\sim 10^{-3} \mathrm{~m} \mathrm{~s}^{-1}$ representing $\sigma_{u} \sim 0.025 \mathrm{~m} \mathrm{~s}^{-1}$ ].

Turbulence estimates are differentiated between being in, or out of the convergence zones identified with acoustic backscatter. Averaged quantities for structure function (denoted with \langle\rangle ) are selectively averaged based on the profile categorization (in/out of convergence zone). Therefore, the averaged quantities of 600 $\mathrm{AD} 2 \mathrm{CP}$ velocity profiles (equivalent to $2.5 \mathrm{~min}$ of data) may span as much as $10 \mathrm{~min}$ of continuous data. The choice of 600 profiles is used to balance the statistical stationarity of environmental conditions and robustness of averaged quantities.

\section{f. SWIFT measurements}

A full description of methods for the SWIFT drifter is available in (Thomson 2012), which will be briefly summarized here. Wave spectra $E_{\mathrm{zz}}(f)$ were estimated from acceleration and velocity measurements made by a nine-axis Microstrain 3DM-GX3-35, using the method of Herbers et al. (2012). Significant wave heights were estimated from wave spectra in the common form $H_{s}=4 \sqrt{\int E_{z z}(f) d f}$. TKE dissipation rates were estimated using a one-sided, second-order structure function approach similar to the one described in section $2 c(1)$, with velocity measurements made from an HR (pulse-coherent) Nortek Aquadopp sampling at $4 \mathrm{~Hz}$, with $4-\mathrm{cm}$ bin size. Water temperature and conductivity were measured with an Aanderaa 4319 CT sensor. Measurements (wave, turbulence, drift speed, location, etc.) were taken as averaged quantities over 8.5-min bursts (with six bursts recorded every hour).

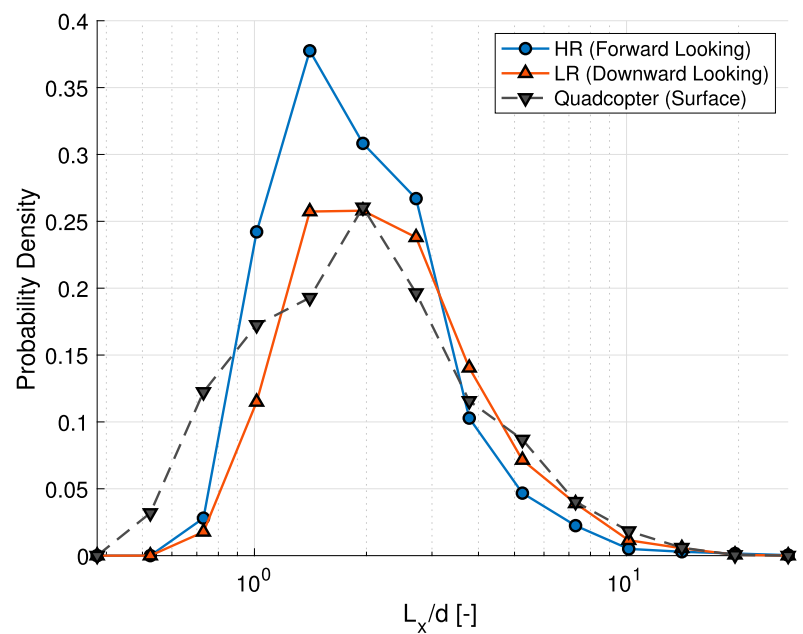

FIG. 4. Distributions of the bubble streak spacing normalized by water depth estimated from the forward looking (HR) AD2CP (blue), the down-looking (LR) AD2CP (orange), and from the quadcopter video (dashed dark gray). Data from the quadcopter are only reported for 8 and 9 Mar (as mentioned in section 2), while HR and LR data are from all deployments where bubble streaks were present (i.e., not 7 Mar).

\section{Results}

\section{a. Geometry of circulations}

Figure 4 shows estimates of the spacing between windrows $L_{x}$ normalized by water depth. The spacing between windrows represents twice the size of a single wind-aligned vortex. Therefore, normalized spacing $L_{x} / d=2$ implies either full depth vortex pairs with an aspect ratio of 1 , or nonunity aspect ratio. The spacing of convergence zones in $\mathrm{AD} 2 \mathrm{CP}$ backscatter was qualitatively consistent with surface bubbles identified with the GoPro (e.g., Figs. 3c and 3d), however a more rigorous evaluation was not done due to the lack of measured camera pose (making it difficult to accurately align the two spatiotemporal signals). Dominant cell spacing from the quadcopter on 8 and 9 March were in agreement with nearby cell spacing estimated with the HR AD2CP. The three distributions of $L_{x} / d$ shown in Fig. 4 are similar, even though they represent differences in measurement depth (quadcopter imagery representing the surface, HR depth approximately $z=0.8 \mathrm{~m}$, and LR depth approximately $z=1.5 \mathrm{~m}$ ). This implies that the distribution of convergences is consistent from the surface to $\sim 1.5$ - $\mathrm{m}$ water depth.

The windrow spacing measured in this study compare favorably with previous measurements of evolving windrows in deep water. For example, (Plueddemann et al. 1996) reports windrow spacing between 4 and $6 \mathrm{~m}$, with bubble plume depths of 2-6m for wind speeds of $8 \mathrm{~m} \mathrm{~s}^{-1}$ (i.e., Figs. 5 and 6 in Plueddemann et al. 1996), which are 
similar to the spacing observed here over similar wind speeds. However, in this study the growth of waves and circulation cells were limited by fetch, while the deepwater measurements of Plueddemann et al. (1996) spanned growing (duration limited), fully developed, and decaying conditions. Studies of Langmuir cells in shallow water have reported that depth limitations can modify cell spacing, with values of $3<L_{x} / d<10$, compared with $L_{x} / L_{z}=2$ reported for deeper conditions (Gargett and Wells 2007; Thorpe 2004). Here because of the limited fetch, the waves had large values of $k h$ such that deep water geometry might be expected. Last, we note that failure of the peak finding algorithm to identify convergences would result in overestimates at integer multiples of the dominant size, which would result in a bias near $L_{x} / d \approx 4$, unrelated to physical effects.

\section{b. Mean near-surface circulation}

Conditionally averaged near-surface velocities in the spanwise (cross-windrow) direction (Fig. 5) show spanwise convergence and enhanced downward and streamwise (along-windrow) velocities associated with the windrows (at normalized spanwise distances of $-1,0$, 1). Halfway between windrows (normalized spanwise distances of $-0.5,0.5)$ spanwise velocities are near zero, streamwise velocities are smaller than average, and vertical velocities are moderate and upward. Therefore, these near-surface (depths $0.3-0.8 \mathrm{~m}$ ) velocities are qualitatively consistent with counterrotating streamwise vorticity cells, which create a similar velocity field at the surface. Velocity magnitudes scaled by the water-side friction velocity show on average, streamwise and downward velocity scales of $(3 / 2) u_{*}$ and $u_{*}$, respectively, while spanwise velocities scale at $(1 / 2) u *$. Given the extensive averaging, smoothing, and uncertainties in instrument orientation relative to the windrow direction, the spanwise gradients and maximum scaled velocities presented in Fig. 5 may be underestimated. For example, individual transects showed cross-windrow averaged velocity scales of $0.05 \mathrm{~m} \mathrm{~s}^{-1}$ or $5 u *$. Previous studies have suggested spanwise velocities scale as $\sqrt{u_{*} U_{s 0}}$ (Plueddemann et al. 1996),down which would predict slightly larger velocities on average, but are consistent with a few individual transect averages.

In addition to the velocity variations consistent with counterrotating vortex pair, there appear to be secondary peaks in the vertical velocity at normalized spanwise coordinates $\pm 0.25, \pm 0.75$. These secondary peaks could be artifacts caused by sampling issues, such as beam spreading across vertical gradients, or failure to detect windrows in the acoustic backscatter. It is also possible

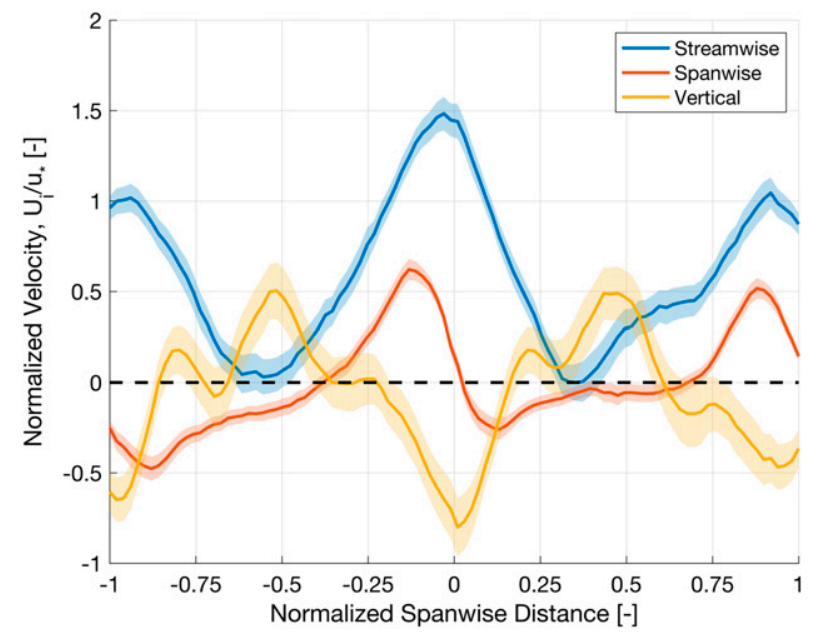

FIG. 5. Velocity components in the streamwise (downwind positive, shown in blue), spanwise (shown in orange), and vertical (shown in yellow, positive up) are normalized by the water-side friction velocity, and shown in the normalized spanwise (crossstreak) direction. Here, spanwise distances are normalized such that windrows (bubbles) would be located at distances of $-1,0$, and 1. The data shown are an average from all transects on days where bubble streaks were seen (all days except 7 Mar 2018). The data are averaged across all horizontal and upward slanted beams, and therefore smooth over any gradients that may exist in the upper $0.3-0.8 \mathrm{~m}$ of the water column. Shaded regions show $95 \%$ confidence intervals for the mean defined as twice the standard error. These error bars therefore indicate the statistical variation from averaging, but do not include errors in estimates of $u *$, windrow location, and $\mathrm{AD} 2 \mathrm{CP}$ orientation.

that the estimates of vertical velocity, which span depths closer to the surface, are capturing smaller cells that do not show up in the spanwise and streamwise components measured at $0.8-\mathrm{m}$ depth.

\section{c. Turbulence measurements}

Experiment averaged, measured structure functions $D_{\text {meas }}(r)$ with the noise $N(r)$ and wave/rotational offsets $r^{2} B(r)$ removed are shown in Fig. 6a, plotted against separation distances $d r$ for the in-convergence and outof-convergence data. Both in-convergence data and outof-convergence data exhibit the $d r^{2 / 3}$ slope characteristic of the inertial subrange. At the largest spatial lags $(d r \sim$ $1.5 \mathrm{~m}$ ) the $d r^{2 / 3}$ slope rolls off, indicating either the importance of waves and rotational motions or a limiting turbulent length scale. This roll-off is larger than the $d r<$ 1.2 used in estimating $\varepsilon$. The in-convergence structure functions are larger than the out-of-convergence data, suggesting an experiment average enhancement in TKE dissipation rates. Normalized noise offsets $N /(\varepsilon d r)^{2 / 3}$ for in convergence and out-of-convergence data shown in Fig. 6b have similar distributions, suggesting that the differences in structure functions is not explained by differences in noise. 

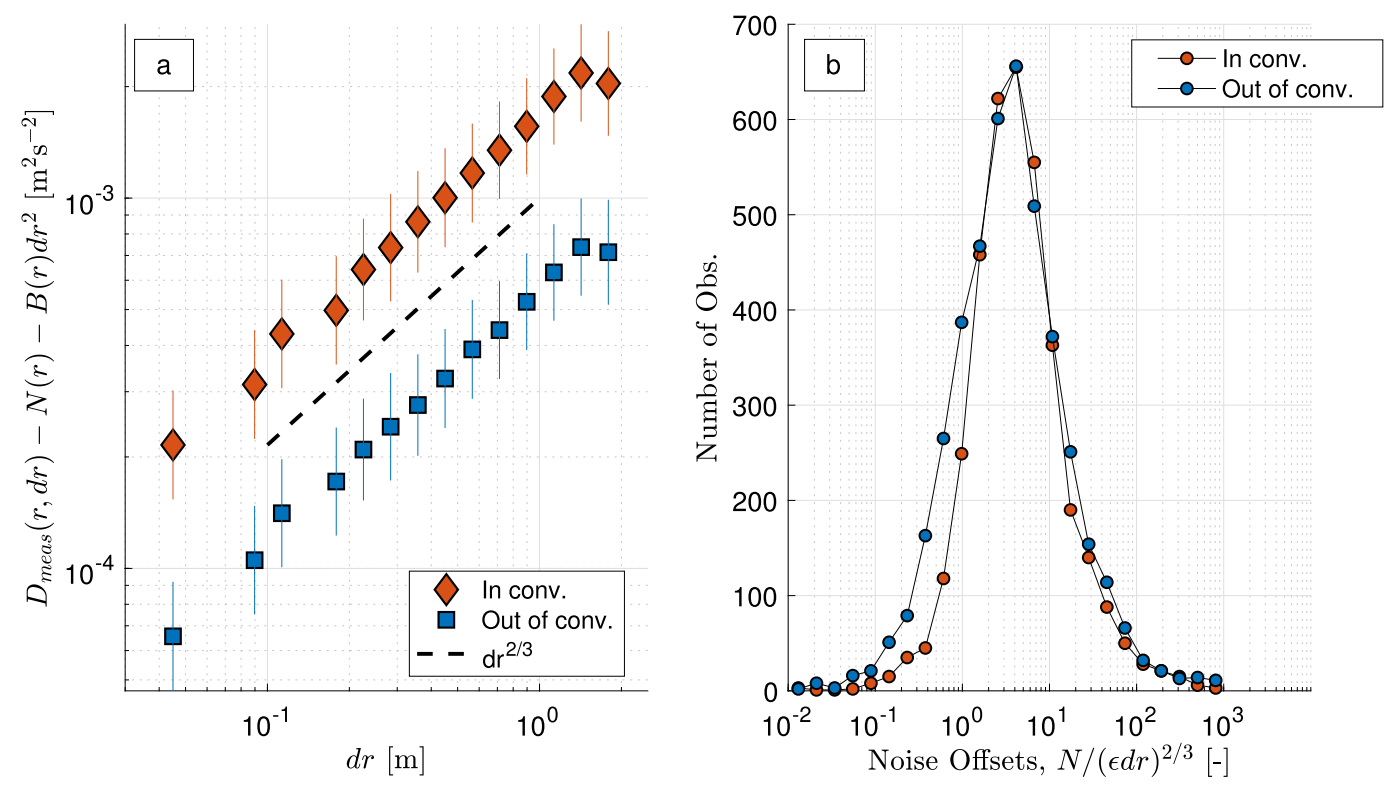

FIG. 6. (a) Measured second-order structure functions $D_{\text {meas }}(r, d r)$, with the best fit noise $N(r)$ and wave/rotational bias $B(r)$ removed, are bin averaged over the experiment and plotted against bin separation distance $d r$ for the in-convergence data (orange diamonds) and the out-of-convergence data (blue squares). Vertical bars represent the $99 \%$ confidence intervals from the mean ( $95 \%$ confidence intervals are smaller than the symbols). Bin edges were chosen arbitrarily, with 20 bins evenly distributed in $\log$ space between $d r=10^{-1.5}$ and $d r=10^{0.5}$, and a minimum of 35 points required for an average (also taken in log space). The dashed black line shows the expected inertial subrange slope $d r^{2 / 3}$. (b) A histogram shows the normalized noise offsets estimated by the in-convergence (orange), and out-of-convergence (blue) data.

TKE dissipation rates estimated from zodiac measurements and from the SWIFT drifter on 9 March are shown in Fig. 7. On this day, short wave breaking was common and bubbles accumulated into along-wind streaks. Turbulence estimates under windrows are larger than estimates outside of windrows, although this effect is primarily limited to the measurements closer to the surface (from the upward slanted, and three horizontal beams). TKE dissipation rates estimated from the zodiac within the convergence zones agree well with SWIFT data. On other deployment days, SWIFT turbulence measurements were similar, or exceeded zodiac estimates from within the convergence zones (not shown in Fig. 7, but all deployment days are used in subsequent analysis).

\section{d. Scaled turbulence}

Measured TKE dissipation rates mostly exceed values predicted by the surface and bottom boundary law of the wall. This holds for data both in and out of the convergence zones, and for zodiac-derived and SWIFT-derived values, as shown in Fig. 8. The only exceptions are the out-of-streak data lowest in the water column which approach wall scalings. These enhanced turbulent values are consistent with previous studies of surface boundary layers in deep water (Agrawal et al. 1992; Terray et al. 1996; Sutherland and Melville 2015; Thomson et al.
2016) and results in shallower environments (Jones and Monismith 2008). Mean tidal velocities were small during the deployments, and measurements were mostly contained in the top half of the water column where bottom boundary layer effects were expected to be small, as shown in Fig. 8b. Measurements

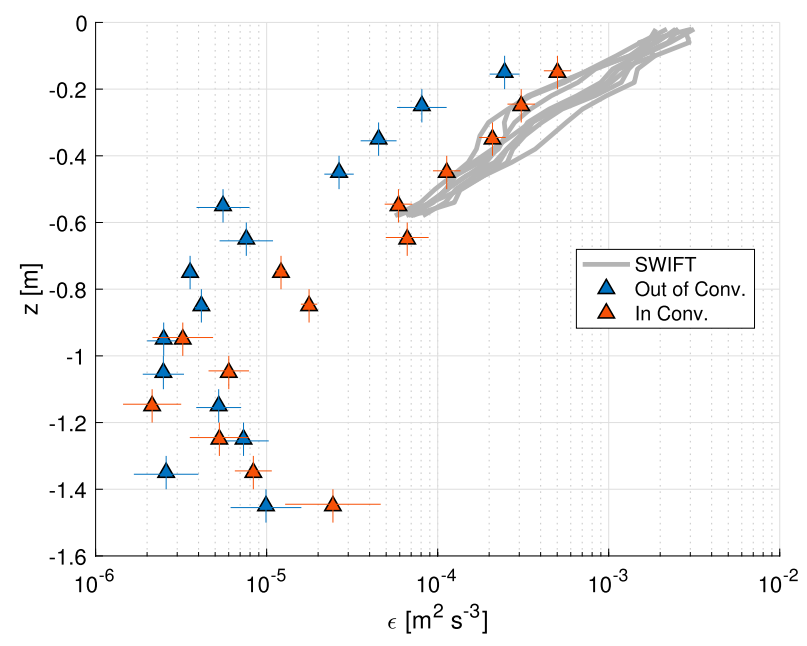

FIG. 7. TKE dissipation rates are shown from 9 Mar 2018. Zodiacderived dissipations are estimated with equivalent 2.5-min time for profiles in (orange) and out (blue) of the convergence zones, and averaged for the deployment. SWIFT-derived dissipations are estimated as an average over $10 \mathrm{~min}$ of sampling (gray lines). 


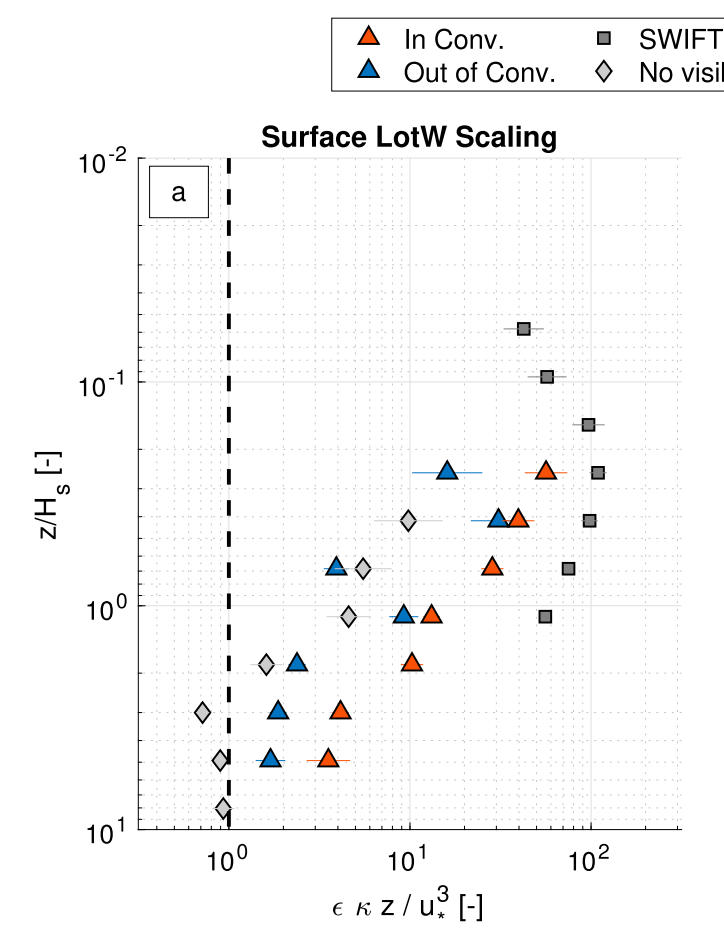

$$
\text { breaking }- \text { Law of the Wall }
$$

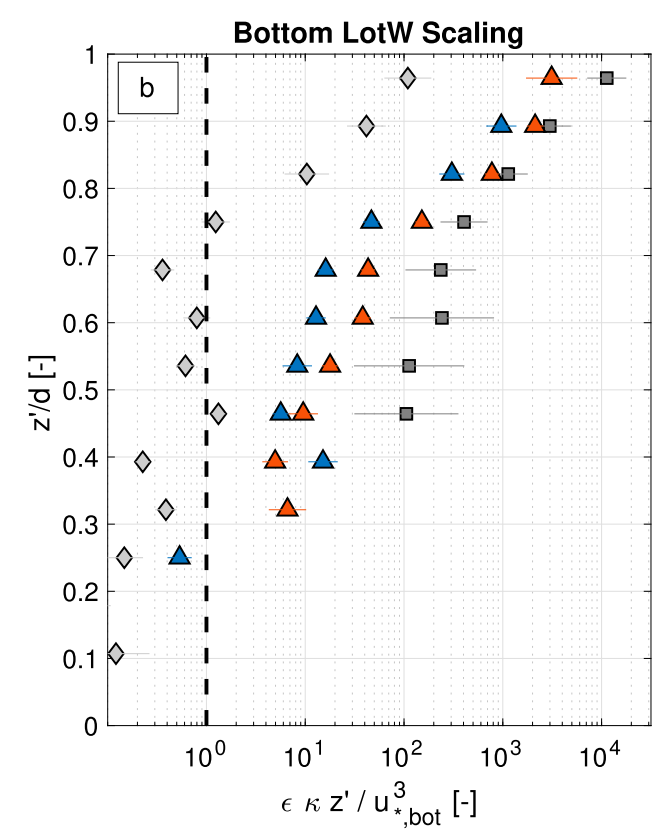

FIG. 8. Turbulence data scaled for the (a) surface boundary layer law of the wall (LotW) and (b) the bottom boundary layer law of the wall. Estimates of TKE dissipation rate $\varepsilon$ are normalized by the closest collocated, cotemporal SWIFT measurements of wind input and $H_{s}$, and vertically bin-averaged. Horizontal error bars show the $95 \%$ confidence for the mean in each bin. Vertical dashed lines represent scaled turbulence levels expected from a surface law of the wall in (a) and a bottom boundary law of the wall in (b). Gray diamonds show data from 7 Mar, where wave breaking and windrows were not observed.

of scaled TKE dissipation rate are also shown for the 7 March data, where no air-entraining breakers were seen by eye, and streaks were not visible in the AD2CP backscatter data. For these data, the surface and bottom wall scalings fit the data well, with the exception of measurements less than a wave height $\left(z / H_{s}<1\right)$. It is possible that microbreaking, which is typically not visible by eye (Jessup and Phadnis 2005; Sutherland and Melville 2013), could account for these elevated nearsurface dissipation rates. This would be consistent with Sutherland and Melville (2015), where it was suggested that microbreaking could account for a large fraction of the wave-breaking surface flux.

Turbulence data are compared with the breaking scaling proposed by Craig and Banner (1994) and Terray et al. (1996), as seen in Fig. 9. Here, the Terray et al. (1996) scaling uses constants $A=0.3$ and $\lambda=-2$ [Eq. (1)], and the Craig and Banner (1994) scaling uses constants $z_{0}=0.3 H_{s}, A=3.7$, and $\lambda=-2.7$ [Eq. (2)]. All data (in-convergence, out-of-convergence, and SWIFT) exhibit the characteristic $\left(z / H_{s}\right)^{-2}$ slope at relative depths $0.3<z / H_{s}<4$. Data out of convergence zones at larger depths start to deviate from the $\lambda=-2$ power law slope, which is consistent with those data being closer to the wall scaling shown in Fig. 8. Data do not match with the constant dissipation layer proposed in (Terray et al. 1996) for $z / H_{s}<0.6$, which is consistent with many recent studies that reference data to the free surface (rather than the mean surface) (Gemmrich 2010; Sutherland and Melville 2015; Thomson et al. 2016; Wang and Liao 2016; Zippel et al. 2018). Reasonable agreement is seen with the measured data here and data from Sutherland and Melville (2015) with young waves (wave age $c_{m} / u_{*}<35$ ).

The enhancement of turbulence within the convergences implied by the zodiac data (as shown in Fig. 10) is nearly an order of magnitude larger than that outside the convergences. This enhancement is seen near the surface $(z<1 \mathrm{~m})$ on the daily averaged data (Fig. 10), which has contributions from four independent beams, (the upward slanted, and three horizontal AD2CP beams). The ratio of nondimensional dissipation (scaled as in Fig. 9) also shows the enhancement in $z / H_{s}$. For most of the binned data, the enhancement exceeds the estimated uncertainty. This enhancement is generally largest in the range $z / H_{s}<1$, and decreases as $z / H_{s} \rightarrow 10$ where log layer scaling is expected. These results are consistent with the measurements reported in Thorpe et al. (2003), where dissipation 


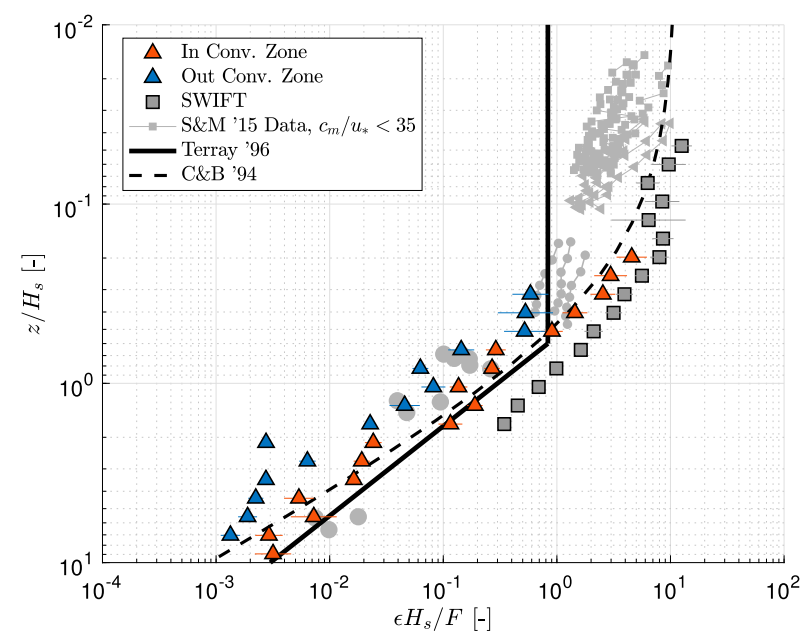

FIG. 9. Scaled turbulence data are shown for the in-convergence (orange), out-of-convergence (blue) zodiac data (triangles), and SWIFT (dark gray squares), with the young wave $\left(c_{m} / u_{*}<35\right)$ data from Sutherland and Melville (2015) shown in light gray (same symbols as originally printed). The scaling suggested in Terray et al. (1996) is shown with the solid black line, and the Craig and Banner (1994) scaling is shown with the dashed black line. Horizontal bars represent $95 \%$ confidence intervals for the mean in each vertical bin average. The 30 bin edges are uniformly distributed in log space between $10^{-2}<z / H_{s}<10^{1}$, and an arbitrary minimum of 30 data points was imposed for displaying a bin average.

rates in Langmuir cells were measured to be between 1.2 and 2.3 times the average value for $2<z / H_{s}<12$. These values are within the confidence values of reported TKE enhancement over similar depth scales in this study, however measurements closer to the surface reported here $\left(z / H_{s}<2\right)$ indicate much larger enhancement.

\section{Discussion}

The young, fetch-limited waves primarily seen during this study are typically associated with larger values of $\mathrm{La}_{t}$, which would imply a shear dominated forcing (compared with the wave dominated forcing of strong Langmuir turbulence). Previous studies have commented that Couette flow can cause circulations that look similar to Langmuir cells (i.e., convergences with spacing of $L / d=2$ ) when $\mathrm{La}_{t}>1$ (Gargett and Wells 2007; Tejada-Martínez and Grosch 2007). Moreover, Couette flow created in laboratories and modeled with LES can create streaks. Although turbulent Langmuir numbers $\mathrm{La}_{t}$ were typically between 0.5 and 0.6 for this study, this value is sensitive to the high frequency portion of the wave spectrum and may be biased low. However, the TKE dissipation rates measured in this study are not consistent with the law of the wall dissipation rates that would be expected in Couette flow (Fig. 8). Therefore, we find it unlikely that the windrows observed in this study are the result of shear-driven Couette flow, and suggest that the windrows are likely related to the surface wave effects that control the TKE dissipation rates.

Convergences can also occur as the result of buoyant overturning at the surface. Although the estimates of heat flux are rough (see methods), we estimate the combined latent and sensible heat flux were in the range $|Q|<30 \mathrm{~W} \mathrm{~m}^{-2}$. Using $|Q|=30 \mathrm{~W} \mathrm{~m}^{-2}$ we estimate that $|\mathrm{Ho}| \sim 10^{-3}$, which is too small to be significant in this dataset. The estimates of $Q$ may be biased due to platform motion, however the size of these errors would not be large enough to significantly modify Ho. The small value of $|\mathrm{Ho}|$ is likely due to a combination of small $Q$ and relatively large $k_{p}$ when compared to other datasets.

Stress generated due to tidal flow over the bottom boundary also can generate shear in shallow water. Gargett and Grosch (2014) suggested a bottom Langmuir number $\mathrm{La}_{H}=u_{* \text {,bot }} / U_{s}$, and that bottom stress becomes relevant for $\mathrm{La}_{H} \sim 2 \mathrm{La}_{t}^{2}$. As noted in Gargett and Grosch (2014), a significant pressure gradient would cause $\mathrm{La}_{H} \gg 1$, while no pressure gradient would cause $\mathrm{La}_{H} \rightarrow \mathrm{La}_{t}^{2}$, as the bottom stress would be forced by the surface. Therefore, the ratio of bottom and surface stress becomes the relevant parameter. During the days where wave breaking and windrows were observed, we estimate $0.1<\mathrm{La}_{H}<0.35$, which implies bottom stress was likely not important. However, on $7 \mathrm{March}$, when no active breaking or windrows were observed, we estimate $\mathrm{La}_{H} \sim 2 \mathrm{La}_{t}^{2}$ which suggests the bottom shear layer may have been important on that day.

\section{a. Impact of observational technique}

Regardless of the mechanism of enhanced TKE dissipation rate within the convergence zones, the implication remains that buoyant drifters, which become trapped in convergence zones, may not measure dissipation rates representative of the spatial surface layer average. This does not negate results reported from drifters in the past, but highlights the care that must be made in interpreting results when convergences are present. The SWIFT dissipation rate measurements in this study were typically larger than the estimates from the zodiac in the convergence zone, which may be due to poor localization in the zodiac data. That is to say, the SWIFT dissipation rate measurements are well localized to be within the convergence zones due to the nature of the drifter and the near vertical beam angle, while the zodiac estimates are less well constrained horizontally due to a shallow beam angle. This implies that the enhancement inside these regions may be larger than suggested from the zodiac measurements alone (e.g., Fig. 10b, SWIFT squares). 

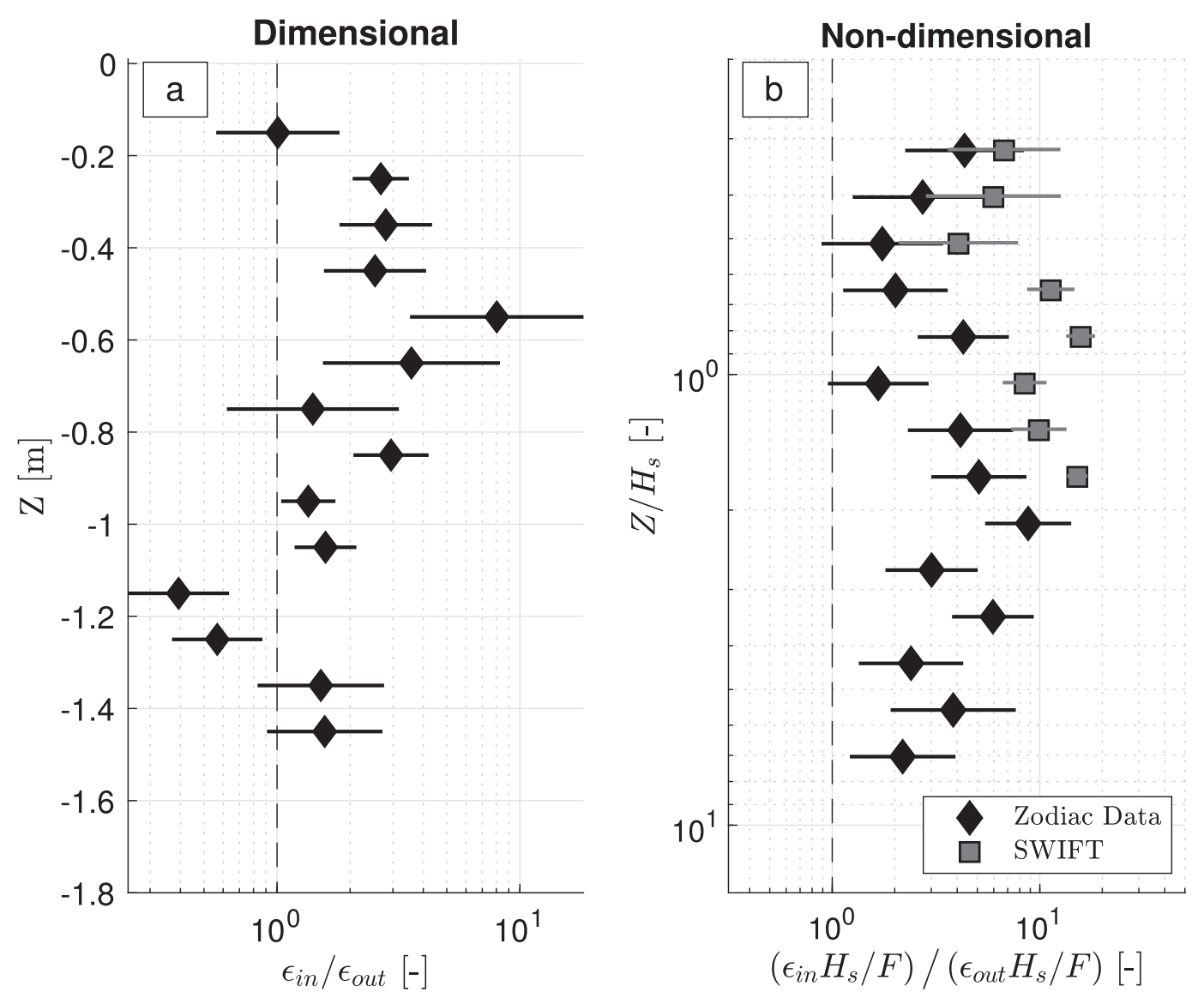

FIG. 10. (a) The ratio of daily averaged TKE dissipation rates are plotted against dimensional depth. Total average values (black) are weighted based on the variance and number of samples for each deployment day. (b) The ratio of nondimensional in-streak to out-of-streak data. Diamonds show zodiac estimates, and squares show the ratio of SWIFT measurements to out-of-convergence, zodiac structure function estimates.

The effects of strong gradients in turbulence at scales smaller than the instrument beam length on the total estimated TKE dissipation rate are unclear and may require further study. The spacing of windrows was typically greater than $6 \mathrm{~m}$ (e.g., Table 1$)$ when compared with the 2-m acoustic beam length, and a maximum separation distance of $d r_{\max }=1.2 \mathrm{~m}$, such that the TKE dissipation rate estimates span approximately $1 / 3$ the length scale of a single vortex. Therefore, it is likely that the enhancement of TKE dissipation rate measured in this study is closer to a minimum enhancement rather than an average enhancement due to the spatial constraints of beam length.

\section{b. Causes for the $\varepsilon$ enhancement}

The enhancement of TKE dissipation in the convergence zones has a number of possible explanations. Thorpe et al. (2003) argued for horizontal advection, noting that bubbles likely act as a localized tracer for wave-breaking turbulence, and that a previous study showed no preferential breaking in regions with bubble convergence (Thorpe 1992). Therefore, randomly distributed patches of enhanced turbulence could be advected into the regions of convergence with the bubbles. In fact, this advection of turbulence has been seen in both upwelling and downwelling regions of Langmuir turbulence in a recent LES study (Kukulka and Veron 2019). However, as mentioned by Thorpe et al. (2003), small scale turbulence can decay rapidly. Direct estimates of advective TKE fluxes were not possible in this dataset, therefore we will assess the relative contributions of these terms using time scales. Here we define the horizontal advection and dissipation time scales as

$$
\begin{gathered}
T_{\mathrm{adv}} \equiv \frac{L_{x}}{2 U_{\mathrm{La}}}, \\
T_{\varepsilon} \equiv \frac{3 u_{*}^{2}}{2 \varepsilon},
\end{gathered}
$$



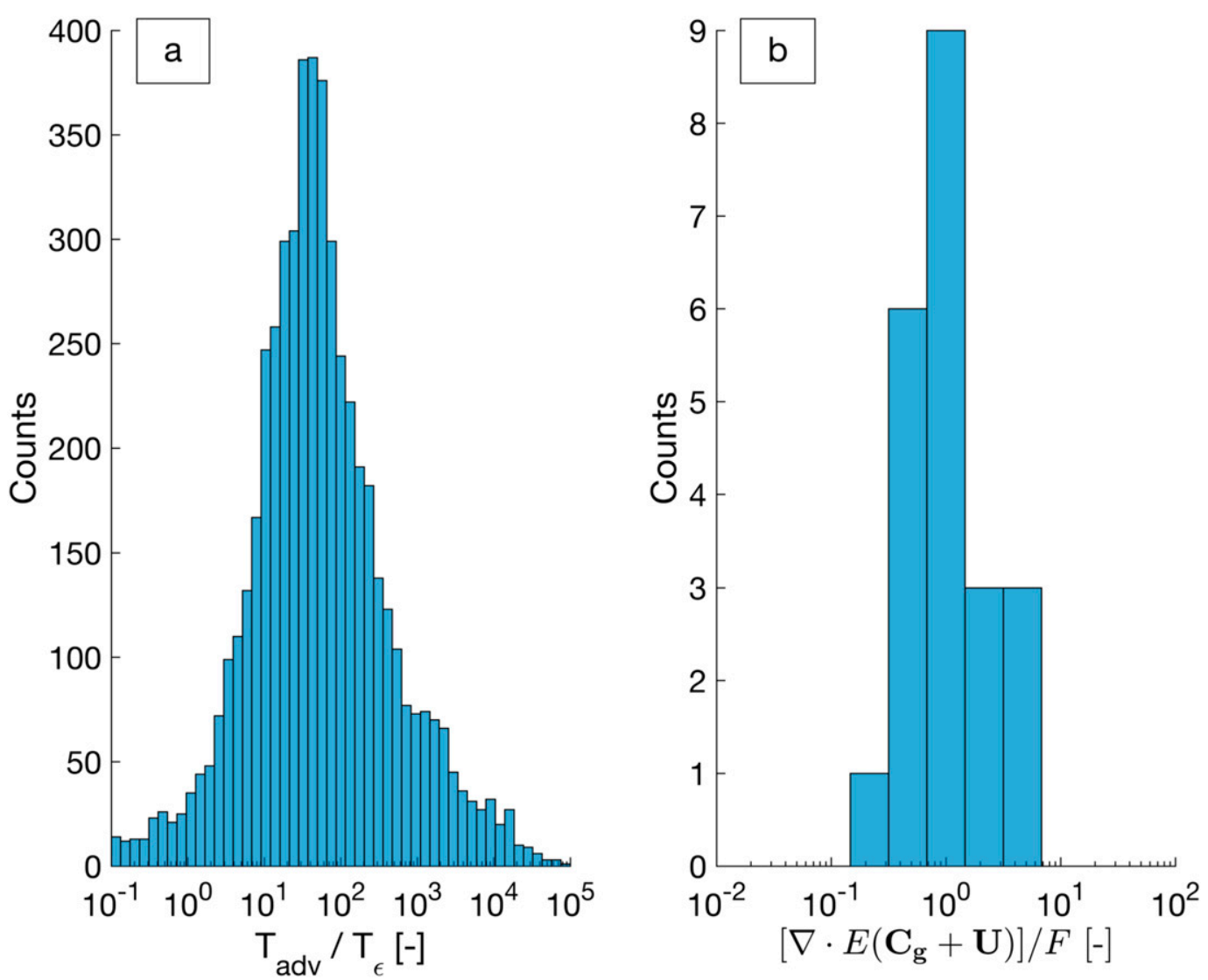

FIG. 11. (a) A histogram of the ratio of time scales for advection and dissipation rate of TKE. Here, the distribution of values tends to be between $10^{1}$ and $10^{2}$, suggesting the time over which horizontal advection takes place is much slower than that for dissipation. The smaller, out-of-convergence dissipation rates (also shown in blue triangles in Fig. 9) are used to estimate time scales. (b) The ratio of wave energy advective flux to the surface flux of TKE, $\nabla \cdot E\left(\mathbf{C}_{\mathbf{g}}+\mathbf{U}\right) / F$, estimated as $2 g H_{s}^{2} /\left(16 c_{\text {eff }} u_{*} L_{x}\right)$, is shown. Fewer counts are seen because estimates are referenced to the SWIFT data, which is averaged on coarser time scales.

where $U_{\mathrm{La}}$ is the horizontal velocity scale of the convergence, and $L_{x}$ is the spacing between convergence zones, and the scale for TKE is assumed to be $(3 / 2) u_{*}^{2}$. Plueddemann et al. (1996) suggested $U_{\mathrm{La}}$ scales as $\left(u_{*} U_{s}\right)^{1 / 2}$, which can be rewritten $u_{*} \mathrm{La}_{t}^{-1}$, or given the small variation of $\mathrm{La}_{t}$ in this study, $\sim 2 u_{*}$. This scaling for $U_{\mathrm{La}}$ is therefore larger than experiment averaged spanwise velocities shown in Fig. 5 (which suggests $U_{\mathrm{La}} \sim 0.5 u_{*}$ ), although we note individual transects showed spanwise velocities up to $U_{\mathrm{La}}=5 u_{*}$, and that the velocity scales shown in Fig. 5 are likely biased low (as mentioned in section 2). A histogram of the ratio of time scales using the out-of-convergence dissipation values at all measured depths, and the larger estimate of $U_{\mathrm{La}}=5 u_{*}$ is used in Fig. 11 to show the distribution of the estimated time scale ratios,

$$
\frac{T_{\mathrm{adv}}}{T_{\varepsilon}}=\frac{L_{x} \varepsilon}{15 u_{*}^{3}} .
$$

The majority of values fall between $10^{1}$ and $10^{2}$, and the distribution has a geometric mean of $\sim 50$, suggesting the time scale of advection is at least an order of magnitude larger than the time scale of dissipation. This suggests that the measured turbulence enhancement is not explained by horizontal advection.

Downward velocities in the convergence zones along with a vertical TKE gradient may also enhance TKE dissipation rates at depth. The vertical advection time scale would be $d / W_{\mathrm{La}}$, where $W_{\mathrm{La}}$ is a vertical velocity scale of the convergence. Mean downward velocities for this dataset scaled as $\sim u_{*}$, with maximum values observed up to $5 u_{*}$. Given the typical aspect ratio for circulations $L_{x} / d \sim 2$, the vertical advective time scale should be similar to the horizontal time scale ratio shown in Fig. 11 (although it would be more appropriate to use the larger, in-convergence dissipation rates, which would increase this time scale ratio by a factor of 2-10). Therefore, it is also unlikely that vertical transport of 
TKE is significantly modifying the spatial distribution of turbulence. Further, downgradient advection would likely decrease the decay slope of $\varepsilon$, which is relatively similar between in- and out-of-convergence zones in Fig. 9, suggesting that the basic diffusive-dissipative balance associated with breaking holds in both regions.

We hypothesize that variations in the surface flux of TKE, $F$, provide a plausible explanation for enhanced dissipations in convergence zones. This contradicts previous studies, where preferential wave breaking was ruled out as a candidate for the formation mechanism of the bubble streaks (Thorpe and Hall 1980; Leibovich 1983). However, we note that these previous studies probably did not resolve the small-scale, microbreaking with their sonar. For waves traveling parallel to the orientation of windrows, convergent spanwise velocities could enhance wave heights (and thus steepness and breaking) in these regions due to the convergence of wave rays. This convergence can be expressed through the advective term in the radiative transfer equation $\nabla \cdot E\left(\mathbf{C}_{g}+\mathbf{U}\right)$, where convergences act to focus wave energy (which cannot propagate downward as currents do). Here, $E=\rho_{w} g H_{s}^{2} / 16$ is the wave energy, and the wave group velocity $\mathbf{C}_{g}$ is assumed to be parallel to the along-windrow direction such that the only spanwise divergence is due to variations in $\mathbf{U}$ (i.e., $d / d x$ terms assumed to be small). The ratio between the magnitude of the spanwise advective wave energy flux,

$$
\left[\nabla \cdot E\left(\mathbf{C}_{g}+\mathbf{U}\right)\right]_{\mathrm{span}} \approx E \frac{d \mathbf{U}}{d y} \sim \frac{d U_{\mathrm{La}}}{d y} \frac{1}{16} g H_{s}^{2},
$$

to the surface flux $F=c_{\text {eff }} u_{*}^{2}$ gives

$$
\frac{2 g H_{s}^{2}}{16 c_{\text {eff }} u_{*} L_{x}},
$$

where we have used the smaller scaling $d U_{\mathrm{La}} / d y \sim 2 u_{*} / L_{x}$, rather than the larger $d U_{\mathrm{La}} / d y \sim 10 u_{*} / L_{x}$ (i.e., $U_{\mathrm{La}} \sim 5 u_{*}$ used in the advective time scale) to highlight that this scaling is robust to even in the lower range of parameter space in this project. We estimate the ratio of the spanwise flux of wave convergence to surface wind flux to be on the order of $10^{-1}-10^{1}$ for this study (Fig. 11b), suggesting that the convergence in wave energy (rather than turbulence) could result in variations of the surface turbulence that are of the same order as the surface flux, $F$.

The near-surface turbulence results presented here agree with some aspects of the studies of (Thorpe et al. 2003; Gemmrich 2012), and conflict with other aspects. The results here are similar to Thorpe et al. (2003) in that dissipation rates are enhanced in regions of convergence where bubbles accumulate. However, contrary to their study, we find TKE dissipation rates are enhanced relative to law of the wall levels (e.g., Fig. 8). Gemmrich (2012) measured similar enhanced near-surface dissipation rates. However, they found dissipation rates were suppressed by bubbles, which was well explained by bubble buoyancy. Their suggested schematic (i.e., Gemmrich 2012, Fig. 4) therefore contrasts with the results of this study, both in the regions of enhancement (in/out-of-convergence), and with respect to law of the wall scaling. Bubbles can act both as a buoyant suppressor of turbulence, as in (Gemmrich 2012), and as a source of turbulence through bubble wakes (Lance and Bataille 1991; Derakhti and Kirby 2014), and future studies are needed to better characterize the influence of bubbles on near-surface turbulence.

\section{Summary}

Measurements of waves, currents, and turbulence were made in a shallow, fetch-limited bay under moderate offshore wind forcing that resulted in wave breaking, and the formation of coherent structures that manifested as wind-aligned bands of bubbles on the surface (windrows). These measurements demonstrate the following:

- The geometry of windrows relative to water depth implies a circulation aspect ratio of one. Conditionally averaged velocities associated with the windrows were consistent with circulation from counterrotating streamwise (roughly wind-aligned) vortex pairs, with velocity scales on the order of $u_{*}$ (Figs. 4 and 5).

- TKE dissipation rates in regions of convergence were estimated to be 2-10 times larger than dissipation rates outside of the convergences. The vertical structure of the turbulence is similar in both cases, and is consistent with the vertical distribution suggested in analytical models and field observations of turbulence under breaking waves (Fig. 9).

- Turbulence likely dissipates before it can be advected into the convergences zones, implying that horizontal advection (convergence) of turbulence does not explain the enhanced TKE dissipation rates. The convergence of wave energy in these regions was estimated to be of similar magnitude to the surface flux, and provides a mechanism for spatially variable TKE input (Fig. 11).

This work is motivated by the importance of the ocean surface boundary layer in the transfer of heat, momentum, and gasses between the ocean and atmosphere. The presence of surface waves fundamentally alters this boundary layer in ways that are not yet fully understood. This study highlights how coherent structures can cause 
small-scale spatial variability in the TKE dissipation rate, an important higher-order boundary layer statistic. These results are interesting in the context of previous field measurements of turbulence in windrows, which have shown both enhancement in bubble plumes (Thorpe et al. 2003), and turbulent suppression from bubble buoyancy (Gemmrich 2012). More work is needed to characterize the complex relation between waves, turbulence, and bubbles. Better understanding of these small scale processes (coherent structures) might help explain the large variance often seen in air-sea measurements, and could lead to more accurate ways to parameterize the ocean surface boundary layer in larger scale simulations.

Acknowledgments. Thank you to Fred Marin for invaluable help collecting field data in harsh conditions. Thank you to Jim Thomson for lending the SWIFT drifter used extensively in this study, and Peter Traykovski for lending the HR Signature AD2CP. The data used in this study are available through the Woods Hole Open Access Server (WHOAS). Support for this work was provided by Woods Hole Oceanographic Institution, through the Interdisciplinary Award and the Postdoctoral Scholar Program. This work was also partially supported by the Centre National d'Études Spatiales (CNES) project WAVE-ICE (PS), and the project WAVESCALE under the "Laboratoire d'Excellence" LabexMER (ANR-10LABX-19) co-funded by a grant from the French government under the program "Investissements d'Avenir" (PS). The BicWin experiment during which this study occurred is funded by the MEOPAR Network of Centers of Excellence (DD) and is a contribution to the research program of Québec-Océan.

\section{APPENDIX}

\section{Second-Order Structure Function in Waves}

The second-order structure function yields a nonzero component from surface wave orbital velocities, as they have structure due to vertical decay, and due to the wave phase. The effect of wave orbital vertical decay is studied by Scannell et al. (2017). However, they use an approximation and do not report the full analytic solution for linear waves which includes the phase component. Here, we present an analytic result of using linear wave orbital velocities as the velocity field in the second-order structure function [Eq. (8)]. Using the resulting formula, we find the contamination to our data is small, and thus the $d r^{2}$ dependence at larger ranges is expected to be resulting from nonwave (platform rotational) motions.
Linear wave orbital velocities are expressed as

$$
\begin{gathered}
\tilde{u}_{n}=\omega_{n} A_{n} e^{-k_{n} z} \sin \theta_{n}, \\
\tilde{w}_{n}=-\omega_{n} A_{n} e^{-k_{n} z} \cos \theta_{n},
\end{gathered}
$$

where $\omega_{n}$ is radian frequency, $k_{n}$ is wavenumber, $A_{n}$ is the amplitude, and $\theta_{n}$ is the phase. Here, $n$ is used such that the full velocity field for a wave spectrum can be approximated by summation across $n$ (e.g., $\sum_{n} \omega_{n} A_{n} e^{-k_{n} z} \cos \theta_{n}$ ).

For a simulated ADCP measurement of the linear wave field only, with beam angle from the vertical $\phi$, the measured velocity at depth $z$,

$$
\begin{aligned}
U_{\text {meas }} & =\sum_{n} \tilde{u}_{n} \sin \phi+\tilde{w}_{n} \cos \phi \\
& =\sum_{n} \omega_{n} A_{n} e^{-k_{n} z}\left[\sin \phi \sin \theta_{n}+\cos \phi \cos \theta_{n}\right] .
\end{aligned}
$$

At a second beam location $r+d r$, the change in horizontal and vertical position along the beam are $\Delta x=$ $|d r| \sin \phi$ and $\Delta z=|d r| \cos \phi$, respectively, and the simulated measured velocity is

$$
\begin{aligned}
U_{\text {meas }}= & \sum_{n} \omega_{n} A_{n} e^{-k_{n}(z-\Delta z)}\left[\sin \phi \sin \left(\theta_{n}+k \Delta x\right)\right. \\
& \left.+\cos \phi \cos \left(\theta_{n}+k \Delta x\right)\right] .
\end{aligned}
$$

The wave contribution to the second-order structure function for a linear wave spectrum is then

$$
\begin{aligned}
\tilde{D}(r)= & \left\langle\left(\sum _ { n } \omega _ { n } A _ { n } e ^ { - k _ { n } z } \left\{\operatorname { s i n } \phi \left[\sin \theta_{n}\right.\right.\right.\right. \\
& \left.-e^{k_{n} \Delta z} \sin \left(\theta_{n}+k_{n} \Delta x\right)\right]-\cos \phi\left[\cos \theta_{n}\right. \\
& \left.\left.\left.\left.-e^{k_{n} \Delta z} \cos \left(\theta_{n}+k_{n} \Delta x\right)\right]\right\}\right)^{2}\right\rangle .
\end{aligned}
$$

This can be reduced using the phasor relation for a linear combination of periodic waves of the same frequency $A_{1} \cos \left(w t+\theta_{1}\right)+A_{2} \cos \left(w t+\theta_{2}\right)=A_{3} \cos \left(w t+\theta_{3}\right)$, where $A_{3}^{2}=\left(A_{1} \cos \theta_{1}+A_{2} \cos \theta_{2}\right)^{2}+\left(A_{1} \sin \theta_{1}+A_{2} \sin \theta_{2}\right)^{2}$. This is applied to simplify the terms in the \{\} , such that

$$
\begin{aligned}
{\left[\cos \theta-e^{k \Delta x} \cos (\theta+k \Delta x)\right]=} & {\left[1-2 e^{k \Delta z} \cos k \Delta x+e^{2 k \Delta z}\right]^{1 / 2} } \\
& \times \cos (\theta+\psi), \\
{\left[\sin \theta-e^{k \Delta x} \sin (\theta+k \Delta x)\right]=} & {\left[1-2 e^{k \Delta z} \cos k \Delta x+e^{2 k \Delta z}\right]^{1 / 2} } \\
& \times \sin (\theta+\psi),
\end{aligned}
$$

where $\psi$ is an arbitrary phase offset. The structure function is then 


$$
\begin{aligned}
\tilde{D}(r)= & \left\langle\left[\sum_{n}\left\{\omega_{n} A_{n} e^{-k_{n} z}\left[1-2 e^{k_{n} \Delta z} \cos k_{n} \Delta x+e^{2 k_{n} \Delta z}\right]^{1 / 2}\right\}\right.\right. \\
& \left.\left.\times\left\{\sin \phi \sin \left(\theta_{n}+\psi\right)+\cos \phi \cos \left(\theta_{n}+\psi\right)\right\}\right]^{2}\right\rangle
\end{aligned}
$$

If the waves are assumed to be linear, then under the assumption of orthogonality, the correlation of terms at differing frequency components $n$ (that result from the square of the summation) become zero. The time average of the phase components becomes $1 / 2$, such that the total expression can be written

$$
\tilde{D}(r)=\sum_{n} \frac{1}{2} \omega_{n}^{2} A_{n}^{2} e^{-2 k_{n} z}\left[1-2 e^{k_{n} \Delta z} \cos k_{n} \Delta x+e^{2 k_{n} \Delta z}\right] .
$$

We note that this relation assumes wave components aligned with the beam deflection angle, which can be included into the phase component $\cos k_{n} \Delta x$. If the waves are assumed to be monochromatic, the result is similar to the results of Scannell et al. (2017).

Using a wave spectrum measured by the SWIFT, the wave effect was found to be a negligible contribution the structure function, giving values on the order of $1 \%$ of the measured structure functions or lower.

\section{REFERENCES}

Agrawal, Y., E. A. Terray, M. A. Donelan, P. A. Hwang, A. J. Williams III, W. M. Drennan, K. K. Kahma, and S. A. Krtaigorodskii 1992: Enhanced dissipation of kinetic energy beneath surface waves. Nature, 359, 219-220, https://doi.org/10.1038/359219a0.

Burchard, H., 2001: Simulating the wave-enhanced layer under breaking surface waves with two-equation turbulence models. J. Phys. Oceangr., 31, 3133-3145, https://doi.org/10.1175/15200485(2001)031<3133:STWELU>2.0.CO;2.

Craig, P. D., and M. L. Banner, 1994: Modeling wave-enhanced turbulence in the ocean surface layer. J. Phys. Oceanogr., 24, 2546-2559, https://doi.org/10.1175/1520-0485(1994)024<2546: MWETIT>2.0.CO;2.

Craik, A. D., and S. Leibovich, 1976: A rational model for Langmuir circulations. J. Fluid Mech., 73, 401-426, https://doi.org/10.1017/ S0022112076001420.

Derakhti, M., and J. T. Kirby, 2014: Bubble entrainment and liquidbubble interaction under unsteady breaking waves. J. Fluid Mech., 761, 464-506, https://doi.org/10.1017/jfm.2014.637.

Drennan, W. M., M. A. Donelan, E. A. Terray, and K. B. Katsaros, 1996: Oceanic turbulence dissipation measurements in SWADE. J. Phys. Oceangr., 26, 808-815, https://doi.org/10.1175/15200485(1996)026<0808:OTDMIS > 2.0.CO;2.

Edson, J. B., A. A. Hinton, K. E. Prada, J. E. Hare, and C. W. Fairall, 1998: Direct covariance flux estimates from mobile platforms at sea. J. Atmos. Oceanic Technol., 15, 547-562, https://doi.org/ 10.1175/1520-0426(1998)015<0547:DCFEFM > 2.0.CO;2.

Elfouhaily, T., B. Chapron, K. Katsaros, and D. Vandemark, 1997: A unified directional spectrum for long and short wind-driven waves. J. Geophys. Res., 102, 15 781-15 796, https://doi.org/ 10.1029/97JC00467.

Elgar, S., B. Raubenheimer, and R. T. Guza, 2001: Current meter performance in the surf zone. J. Atmos. Oceanic Technol., 18, 1735-1746, https://doi.org/10.1175/1520-0426(2001)018<1735: CMPITS $>2.0 . \mathrm{CO} ; 2$.

Gargett, A. E., and J. R. Wells, 2007: Langmuir turbulence in shallow water. Part 1. Observations. J. Fluid Mech., 576, 27-61, https://doi.org/10.1017/S0022112006004575.

— - and C. Grosch, 2014: Turbulence process domination under the combined forcings of wind stress, the Langmuir vortex force, and surface cooling. J. Phys. Oceanogr., 44, 44-67, https://doi.org/10.1175/JPO-D-13-021.1.

Gemmrich, J., 2010: Strong turbulence in the wave crest region. J. Phys. Oceanogr., 40, 583-595, https://doi.org/10.1175/ 2009JPO4179.1.

__ 2012: Bubble-induced turbulence suppression in Langmuir circulation. Geophys. Res. Lett., 39, L10604, https://doi.org/ 10.1029/2012GL051691.

, T. Mudge, and V. Polonichko, 1994: On the energy input from wind to surface waves. J. Phys. Oceanogr., 24, 2413-2417, https:// doi.org/10.1175/1520-0485(1994)024<2413:OTEIFW>2.0.CO;2.

Gerbi, G., J. Trowbridge, E. Terray, A. J. Plueddemann, and T. Kukulka, 2009: Observations of turbulence in the ocean surface boundary layer: Energetics and transport. J. Phys. Oceanogr., 39, 1077-1096, https://doi.org/10.1175/2008JPO4044.1.

Hare, J. E., T. Hara, J. B. Edson, and J. M. Wilczak, 1997: A similarity analysis of the structure of airflow over surface waves. J. Phys. Oceanogr., 27, 1018-1037, https://doi.org/10.1175/ 1520-0485(1997)027<1018:ASAOTS > 2.0.CO;2.

Herbers, T. H. C., P. F. Jessen, T. T. Janssen, D. B. Colbert, and J. H. MacMahan, 2012: Observing ocean surface waves with GPS-tracked buoys. J. Atmos. Oceanic Technol., 29, 944-959, https://doi.org/10.1175/JTECH-D-11-00128.1.

Janssen, P. A., 1999: On the effect of ocean waves on the kinetic energy balance and consequences for the inertial dissipation technique. J. Phys. Oceanogr., 29, 530-534, https://doi.org/ 10.1175/1520-0485(1999)029<0530:OTEOOW > 2.0.CO;2.

Jessup, A., and K. Phadnis, 2005: Measurement of the geometric and kinematic properties of microscale breaking waves from infrared imagery using a PIV algorithm. Meas. Sci. Technol., 16, 1961-1969, https://doi.org/10.1088/0957-0233/16/10/011.

Jones, N. L., and S. G. Monismith, 2008: The influence of whitecapping waves on the vertical structure of turbulence in a shallow estuarine embayment. J. Phys. Oceanogr., 38, 15631580, https://doi.org/10.1175/2007JPO3766.1.

Kahma, K. K., and C. J. Calkoen, 1992: Reconciling discrepancies in the observed growth of wind-generated waves. J. Phys. Oceanogr., 22, 1389-1405, https://doi.org/10.1175/ 1520-0485(1992)022<1389:RDITOG >2.0.CO;2.

Kukulka, T., and R. Harcourt, 2017: Influence of stokes drift decay scale on Langmuir turbulence. J. Phys. Oceanogr., 7, https:// doi.org/10.1175/JPO-D-16-0244.1.

— turbulence in the ocean surface boundary layer. J. Phys. Oceanogr., 49, 409-429, https://doi.org/10.1175/JPO-D-18-0081.1.

Lance, M., and J. Bataille, 1991: Turbulence in the liquid phase of a uniform bubbly air-water flow. J. Fluid Mech., 222, 95-118, https://doi.org/10.1017/S0022112091001015.

Langmuir, I., 1938: Surface motion of water induced by wind. Science, 87, 119-123, https://doi.org/10.1126/science.87.2250.119.

Large, W., and S. Pond, 1981: Open ocean momentum flux measurements in moderate to strong winds. J. Phys. Oceanogr., 11, 
324-336, https://doi.org/10.1175/1520-0485(1981)011<0324: OOMFMI $>2.0 . \mathrm{CO} ; 2$.

Leibovich, S., 1983: The form and dynamics of Langmuir circulations. Annu. Rev. Fluid Mech., 15, 391-427, https://doi.org/ 10.1146/annurev.fl.15.010183.002135.

Li, M., and C. Garrett, 1995: Is Langmuir circulation driven by surface waves or surface cooling? J. Phys. Oceanogr., 25, 64-76, https:// doi.org/10.1175/1520-0485(1995)025<0064:ILCDBS>2.0.CO;2.

Liu, H., G. Peters, and T. Foken, 2001: New equations for sonic temperature variance and buoyancy heat flux with an omnidirectional sonic anemometer. Bound.-Layer Meteor., 100, 459-468, https://doi.org/10.1023/A:1019207031397.

McWilliams, J. C., P. P. Sullivan, and C.-H. Moeng, 1997: Langmuir turbulence in the ocean. J. Fluid Mech., 334, 1-30, https:// doi.org/10.1017/S0022112096004375.

Otsu, N., 1979: A threshold selection method from gray-level histograms. IEEE Trans. Syst. Man Cybern., 9, 62-66, https:// doi.org/10.1109/TSMC.1979.4310076.

Plant, N. G., K. T. Holland, and M. C. Haller, 2008: Ocean wavenumber estimation from wave-resolving time series imagery. IEEE Trans. Geosci. Remote Sens., 46, 2644-2658, https:// doi.org/10.1109/TGRS.2008.919821.

Plueddemann, A. J., J. A. Smith, D. M. Farmer, R. A. Weller, W. R. Crawford, R. Pinkel, S. Vagle, and A. Gnanadesikan, 1996: Structure and variability of Langmuir circulation during the surface waves processes program. J. Geophys. Res., 101, 35253543, https://doi.org/10.1029/95JC03282.

Rusello, P. J., 2009: A practical primer for pulse coherent instruments. Tech. Rep. TN-027, Nortek AS, 12 pp.

Scannell, B. D., T. P. Rippeth, J. H. Simpson, J. A. Polton, and J. E. Hopkins, 2017: Correcting surface wave bias in structure function estimates of turbulent kinetic energy dissipation rate. J. Atmos. Oceanic Technol., 34, 2257-2273, https://doi.org/ 10.1175/JTECH-D-17-0059.1.

Scully, M. E., A. W. Fisher, S. E. Suttles, L. P. Sanford, and W. C. Boicourt, 2015: Characterization and modulation of Langmuir circulation in Chesapeake Bay. J. Phys. Oceanogr., 45, 26212639, https://doi.org/10.1175/JPO-D-14-0239.1.

- J. H. Trowbridge, and A. W. Fisher, 2016: Observations of the transfer of energy and momentum to the oceanic surface boundary layer beneath breaking waves. J. Phys. Oceanogr., 46, 1823-1837, https://doi.org/10.1175/JPO-D-15-0165.1.

Shcherbina, A. Y., E. A. D'Asaro, and S. Nylund, 2018: Observing finescale oceanic velocity structure with an autonomous Nortek acoustic Doppler current profiler. J. Atmos. Oceanic Technol., 35, 411-427, https://doi.org/10.1175/JTECH-D-17-0108.1.

Skyllingstad, E. D., and D. W. Denbo, 1995: An ocean large-eddy simulation of Langmuir circulations and convection in the surface mixed layer. J. Geophys. Res., 100, 8501-8522, https:// doi.org/10.1029/94JC03202.

Smith, J. A., 1996: Observations of Langmuir circulation, waves, and the mixed layer. The Air Sea Interface: Radio and Acoustic Sensing, Turbulence, and Wave Dynamics, M. A. Donelan, W. H. Hui, and W. J. Plant, Eds., University of Toronto Press, 613-622 pp.

Sullivan, P. P., J. C. McWilliams, and W. K. Melville, 2007: Surface gravity wave effects in the oceanic boundary layer: Large-eddy simulation with vortex force and stochastic breakers. J. Fluid Mech., 593, 405-452, https://doi.org/10.1017/S002211200700897X.

Sutherland, P., and W. K. Melville, 2013: Field measurements and scaling of ocean surface wave-breaking statistics. Geophys. Res. Lett., 40, 3074-3079, https://doi.org/10.1002/GRL.50584.

- , and —_, 2015: Field measurements of surface and nearsurface turbulence in the presence of breaking waves.
J. Phys. Oceanogr., 45, 943-965, https://doi.org/10.1175/ JPO-D-14-0133.1.

, and D. Dumont, 2018: Marginal ice zone thickness and extent due to wave radiation stress. J. Phys. Oceanogr., 48, 18851901, https://doi.org/10.1175/JPO-D-17-0167.1.

Tejada-Martínez, A., and C. Grosch, 2007: Langmuir turbulence in shallow water. part 2. Large-eddy simulation. J. Fluid Mech., 576, 63-108, https://doi.org/10.1017/S0022112006004587.

Terray, E., M. Donelan, Y. Agrawal, W. Drennan, K. Kahma, A. Williams, P. Hwang, and S. Kitaigorodskii, 1996: Estimates of kinetic energy dissipation under breaking waves. J. Phys. Oceanogr., 26, 792-807, https://doi.org/10.1175/1520-0485(1996) 026<0792:EOKEDU $>2.0 . \mathrm{CO} ; 2$.

Thomson, J., 2012: Wave breaking dissipation observed with SWIFT drifters. J. Atmos. Oceanic Technol., 29, 1866-1882, https://doi.org/10.1175/JTECH-D-12-00018.1.

, B. Polagye, V. Durgesh, and M. C. Richmond, 2012: Measurements of turbulence at two tidal energy sites in Puget Sound, WA. J. Ocean. Eng, 37, 363-374, https://doi.org/10.1109/JOE.2012.2191656.

, E. A. D'Asaro, M. Cronin, E. Rogers, R. Harcourt, and A. Schcerbina, 2013: Waves and the equilibrium range at Ocean Weather Station P. J. Geophys. Res. Oceans, 118, 59515962, https://doi.org/10.1002/2013JC008837.

, M. Schwendeman, S. F. Zippel, S. Moghimi, J. Gemmrich, and W. E. Rogers, 2016: Wave-breaking turbulence in the ocean surface layer. J. Phys. Oceanogr., 46, 1857-1870, https:// doi.org/10.1175/JPO-D-15-0130.1.

Thorpe, S. A., 1992: Bubble clouds and the dynamics of the upper ocean. Quart. J. Roy. Meteor. Soc., 118, 1-22, https://doi.org/ 10.1002/qj.49711850302.

, 2004: Langmuir circulation. Annu. Rev. Fluid Mech., 36, 5579, https://doi.org/10.1146/annurev.fluid.36.052203.071431. , and A. Hall, 1980: The mixing layer of loch ness. J. Fluid Mech., 101, 687-703, https://doi.org/10.1017/S0022112080001875.

T. Osborn, J. Jackson, A. Hall, and R. Lueck, 2003: Measurements of turbulence in the upper-ocean mixing layer using Autosub. J. Phys. Oceanogr., 33, 122-145, https://doi.org/ 10.1175/1520-0485(2003)033<0122:MOTITU>2.0.CO;2.

Umlauf, L., and H. Burchard, 2003: A generic length-scale equation for geophysical turbulence models. J. Mar. Res., 61, 235265, https://doi.org/10.1357/002224003322005087.

Wang, B., and Q. Liao, 2016: Field observations of turbulent dissipation rate profiles immediately below the air-water interface. J. Geophys. Res. Oceans, 121, 4377-4391, https://doi.org/10.1002/2015JC011512.

Wiles, P., T. P. Rippeth, J. Simpson, and P. Hendricks, 2006: A novel technique for measuring the rate of turbulent dissipation in the marine environment. Geophys. Res. Let., 33, L21608, https://doi.org/10.1029/2006GL027050.

Yelland, M., P. Taylor, I. Consterdine, and M. Smith, 1994: The use of the inertial dissipation technique for shipboard wind stress determination. J. Atmos. Oceanic Technol., 11, 1093-1108, https:// doi.org/10.1175/1520-0426(1994)011<1093:TUOTID>2.0.CO;2.

Zedel, L., A. Hay, R. Cabrera, and A. Lohrmann, 1996: Performance of a single-beam pulse-to-pulse coherent doppler profiler. J. Ocean. Eng., 21, 290-297, https://doi.org/10.1109/48.508159.

Zippel, S. F., J. Thomson, and G. Farquharson, 2018: Turbulence from breaking surface waves at a river mouth. J. Phys. Oceanogr., 48, 435-453, https://doi.org/10.1175/JPO-D-17-0122.1.

, T. Maksym, M. E. Scully, P. Sutherland, and D. Dumont, 2019: Observations of turbulence and the geometry and circulation of windrows in a small bay in the St. Lawrence estuary. Woods Hole Open Access Server, accessed 11 November 2019, https://doi.org/10.26025/1912/24792. 